JIRSS (2019)

Vol. 18, No. 01, pp 191-236

DOI: 10.29252/jirss.18.1.191

\title{
Parameter Estimation and Prediction for the Generalized Half- Normal Distribution under Progressive Hybrid Censoring
}

\author{
Farha Sultana $^{1}$, Yogesh Mani Tripathi ${ }^{1}$, and Manoj Kumar Rastogi ${ }^{2}$ \\ ${ }^{1}$ Department of Mathematics, Indian Institute of Technology Patna, Bihta-801106, India. \\ 2 Department of Statistics, Patna University, Patna-800005, India.
}

Received: 01/22/2018, Revision received: 09/01/2018, Published online: 03/28/2019

\begin{abstract}
In this paper, the problem of estimating unknown parameters of a generalized half- normal distribution is considered under Type II progressive hybrid censoring which is a combination of Type II progressive and hybrid censoring schemes. We obtain maximum likelihood estimators of parameters and also construct asymptotic intervals using the observed Fisher information matrix. Further Bayes estimates are computed under the squared error loss function by applying different approximation methods. We also obtain prediction estimates and prediction intervals of censored observations. The performance of different methods is compared using Monte Carlo simulations and a real data set is analyzed for illustrative purposes.
\end{abstract}

Keywords. Bayesian estimates, HPD intervals, Maximum likelihood estimators, Tierney and Kadane method, Type II progressive hybrid censoring, Prediction.

MSC: 62N01; 62N02; 62N05.

Farha Sultana (farha.pma15@iitp.ac.in)

Corresponding Author: Yogesh Mani Tripathi (yogesh@iitp.ac.in)

Manoj Kumar Rastogi (manoj@iitp.ac.in) 


\section{Introduction}

Many life testing experiments, in general, involve making inferences about unknown parameters or reliability characteristics such as failure probabilities in presence of certain censored observations. Censoring is one of the important method which deals with the practical problems of observing data in studies like clinical trials, industrial experiments, mortality analysis, etc. Probably the two most common censoring schemes are Type I and Type II censoring.

Suppose that a total of $n$ test units is initially subjected to a life testing experiment. Then in Type I censoring observations are recorded up to a prespecified time point $T$ and no failures are recorded after $T$. On the other hand in Type II censoring the test continues until a prescribed $r(\leq n)$ number of failure times has been observed. Epstein (1954) introduced the concept of hybrid censoring and studied a one-parameter exponential distribution with unknown mean. In this censoring, a life test continues until a prescribed time point $T$ has reached or a prefixed $r$ number of test units had failed, whichever happens earlier, that is, the test stops at the time point $\min \left(X_{r: n}, T\right)$ where $X_{r: n}$ is the failure time of the $r$ th observation. This is known as a hybrid Type I censoring. Note that in these schemes live test units cannot be removed during the experimentation. Progressive Type II censoring is a more general scheme in this regard where live test units can be removed in between the experiment. Under this censoring, a sample of size $m(\leq n)$ can be obtained as follows. At the first failure time $X_{1: m: n}$, the $R_{1}$ number of surviving units is removed from the experiment at random. Similarly at the second failure time $X_{2: m: n}$, the $R_{2}$ number of surviving units is removed from the experiment again at random. The experiment continues till the $m$ th failure time is observed and by this time all the remaining $R_{m}=n-m-R_{1}-R_{2}-\ldots-R_{m-1}$ test units are removed from the experiment. The number $R_{i}, i=1,2, \ldots, m$ is referred to as the corresponding censoring scheme and is fixed before the start of the experiment.

This censoring has found wide applications in life testing experiments and one may refer to Balakrishnan and Aggarwala (2000), Balakrishnan and Cramer (2014) for a detailed discussion on this topic. In this type of censoring observing a sufficient number of samples can sometimes be time-consuming. Kundu and Joarder (2006) initially discussed Type II progressive hybrid censoring scheme in literature as a mixture of Type II progressive and hybrid censoring schemes. In this case, a life test stops either when a prescribed number of failure times has been recorded or when a prescribed time point has reached. Briefly, it can be described as follows. At the first failure time $X_{1: m: n}$, the $R_{1}$

number of units from the remaining $(n-1)$ surviving units are randomly removed from 
the experiment. Similarly, at the second failure time $X_{2: m: n}$, the $R_{2}$ number of units from the remaining $\left(n-R_{1}-2\right)$ surviving units are randomly removed and the experiment continues. Note that if $m$ th failure occurs before the time $T$ then the test stops at the time point $X_{m: m: n}$. Otherwise, it stops at time $T$ such that $X_{j: m: n}<T<X_{j+1: m: n}$ and by this time all the remaining $R_{j}^{*}=n-R_{1}-\ldots-R_{j}-j$ surviving units are removed. In the second case $j(<m)$ number of observations is recorded up to the time point $T$. Since in Type II progressive hybrid censoring scheme a life test stops at a random time $T_{1}=\min \left(X_{m: m: n}, T\right)$ and so the observed data may be one of the following two types

$$
\left\{\begin{array}{lc}
\text { case } I:\left\{X_{1: m: n}, X_{2: m: n}, \ldots, X_{m: m: n}\right\}, & \text { if } X_{m: m: n}<T \\
\text { case } I I:\left\{X_{1: m: n}, X_{2: m: n}, \ldots, X_{j: m: n}\right\}, & \text { if } X_{j: m: n}<T<X_{j+1: m: n}
\end{array}\right.
$$

Several authors have studied different lifetime models using Type II progressive hybrid censoring. Kundu and Joarder (2006) obtained maximum likelihood and Bayes estimators of the unknown rate parameter and proposed different confidence intervals as well. The authors compared different methods numerically using Monte Carlo simulations and analyzed a real data set for illustrative purposes. Lin et al. (2009) obtained maximum likelihood estimators (MLEs) and approximate maximum likelihood estimators (AMLEs) of the unknown parameters of a Weibull distribution under this censoring scheme. A simulation study is conducted to assess the behavior of proposed methods. Several useful comments are obtained based on this study. Recently, Azizpour and Asgharzadeh (2017) considered the point and interval estimation of unknown parameters of Type II generalized logistic distribution under the assumption that data are observed using Type II hybrid progressive censoring. The authors obtained maximum likelihood and approximate maximum likelihood estimators of unknown location and scale parameters. Asymptotic and bootstrap-p confidence intervals based on these two estimators are discussed as well. A numerical comparison is performed to compare the performance of the proposed methods and two illustrative examples are discussed. One may also refer to Mokhtari et al. (2011), Balakrishnan and Kundu (2013), Kayal et al. (2017) for some more interesting applications of Type II progressive hybrid censoring in life testing experiments.

In this paper, we consider the problem of estimating unknown parameters of a generalized half-normal distribution based on Type II progressive hybrid censoring. We also consider the problem of predicting censored observations under this censoring. The probability density function (PDF) and the cumulative distribution function (CDF) 
are respectively given by

$$
\begin{aligned}
& f_{X}(x ; \alpha, \theta)=\sqrt{\frac{2}{\pi}} \alpha \sqrt{\theta} x^{\alpha-1} \exp \left\{-\frac{1}{2} \theta x^{2 \alpha}\right\}, \quad x \geq 0, \alpha>0, \theta>0, \\
& F_{X}(x ; \alpha, \theta)=1-2 \Phi\left[-\sqrt{\theta} x^{\alpha}\right], \quad x \geq 0,
\end{aligned}
$$

where $\Phi($.$) is the CDF of the standard normal distribution. Note that \alpha$ and $\theta$ respectively denote shape and scale parameter. We denote $\operatorname{GHN}(\alpha, \theta)$ to be the generalized half-normal distribution. Cooray and Ananda (2008) initially studied various probabilistic properties of this distribution.

In particular, the hazard rate function may be monotonic or bathtub shaped depending upon parameter values. The generalized half-normal distribution is treated as an alternative model to lognormal, gamma, Weibull and Birnbaum-Saunders distributions, among others. Ahmadi et al. (2015) also studied this distribution with progressive censoring and obtained various inferences on unknown parameters. Ahmadi et al. (2015) considered estimation of $\alpha$ and $\theta$ under progressive Type I interval censoring. They proposed different estimators of both the unknown parameters and compared their performance numerically using simulations. A real data analysis is also discussed in support of proposed methods.

We mention that though different methods have been discussed for making inference upon unknown parameters of the generalized half-normal distribution under complete and censored sampling situations, however, to the best of our knowledge, a comprehensive comparison of various estimation procedures has not been discussed, in particular under progressive hybrid censoring. We have used an expectationmaximization (EM) algorithm to compute maximum likelihood estimates of $\alpha$ and $\theta$ using progressive hybrid censored samples. Bayes estimates are obtained using two different approaches such as Tierney and Kadane method (see, Tierney and Kadane (1986)) and importance sampling procedure. Importance sampling is further used in the construction of Bayesian credible intervals of unknown parameters. We have compared the performance of different estimation procedures and observe that it is relatively useful for practitioners to make note of better estimation method that may be applied for the generalized half-normal distribution. Another contribution of our work is that we have developed prediction estimates and prediction intervals of censored observations under progressive hybrid censoring which have not been considered before. We have discussed both classical and Bayes approaches to compute predictive estimates. Prediction problems arise quite naturally in many reliability and life testing experiments. 
We have organized the rest of this paper as follows. In Section 2, we discuss the maximum likelihood estimators of $\alpha$ and $\theta$ based on Type II progressive hybrid censored samples. The asymptotic confidence intervals of these parameters are also obtained from the observed Fisher information matrix. In Section 3, Bayes estimators are derived under the squared error loss function using gamma prior distributions. We use two different approximation methods to compute these Bayes estimates. We further construct the highest posterior density (HPD) intervals of unknown parameters of the generalized half-normal distribution. In Section 4, we discuss prediction estimates and prediction intervals of censored observations under the considered censoring scheme. Finally, in Section 5, different estimators are compared using Monte Carlo simulations and analysis of a real data set is also carried out for illustrative purpose. A concluding remark is given in Section 6.

\section{Maximum Likelihood Estimation}

In this section, we derive MLEs of unknown parameters of the $G H N(\alpha ; \theta)$ distribution using Type II progressive hybrid censored samples. Suppose $n$ independent test units with lifetimes following a generalized half normal distribution are subjected to a life test. Then a Type II progressive hybrid censored sample is observed using the given censoring scheme. The corresponding likelihood functions of $\alpha$ and $\theta$ are given by

$$
\left\{\begin{aligned}
\text { case } I: L(\alpha, \theta \mid x) & \propto \prod_{i=1}^{m} f\left(x_{i: m: n}\right)\left[1-F\left(x_{i: m: n}\right)\right]^{R_{i}} \\
\text { case } I I: L(\alpha, \theta \mid x) & \propto \prod_{i=1}^{j} f\left(x_{i: m: n}\right)\left[1-F\left(x_{i: m: n}\right)\right]^{R_{i}}[1-F(T)]^{R_{j}^{*}} .
\end{aligned}\right.
$$

For the considered model these functions can combinedly be expressed as

$$
L(\alpha, \theta \mid x) \propto \alpha^{d} \theta^{\frac{d}{2}} e^{U(\alpha, \theta)} \prod_{i=1}^{d} x_{i}^{\alpha-1},
$$

where $x_{i}=x_{i: m: n}$ with $d$ and $U(\alpha, \theta)$ being defined as, respectively,

$$
d= \begin{cases}m, & \text { for case } I, \\ j, & \text { for case } I I\end{cases}
$$


and

$$
U(\alpha, \theta)= \begin{cases}-\frac{\theta}{2} \sum_{i=1}^{m} x_{i}^{2 \alpha}+\sum_{i=1}^{m} R_{i} \log \left(\Phi\left(-\sqrt{\theta} x_{i}^{\alpha}\right)\right), & \text { for case } I, \\ -\frac{\theta}{2} \sum_{i=1}^{j} x_{i}^{2 \alpha}+\sum_{i=1}^{j} R_{i} \log \left(\Phi\left(-\sqrt{\theta} x_{i}^{\alpha}\right)\right)+R_{j}^{*} \log \left(\Phi\left(-\sqrt{\theta} T^{\alpha}\right)\right), & \text { for case II. }\end{cases}
$$

To obtain MLEs of parameters, we consider the associated log-likelihood function

$$
\log L(\alpha, \theta \mid x) \propto d \log \alpha+\frac{d}{2} \log \theta+U(\alpha, \theta)+(\alpha-1) \sum_{i=1}^{d} \log x_{i}
$$

and then likelihood equations are obtained as

$$
\frac{\partial \log L}{\partial \alpha}=\frac{d}{\alpha}+U_{\alpha}+\sum_{i=1}^{d} \log x_{i}=0
$$

and

$$
\frac{\partial \log L}{\partial \theta}=\frac{d}{2 \theta}+U_{\theta}=0
$$

where,

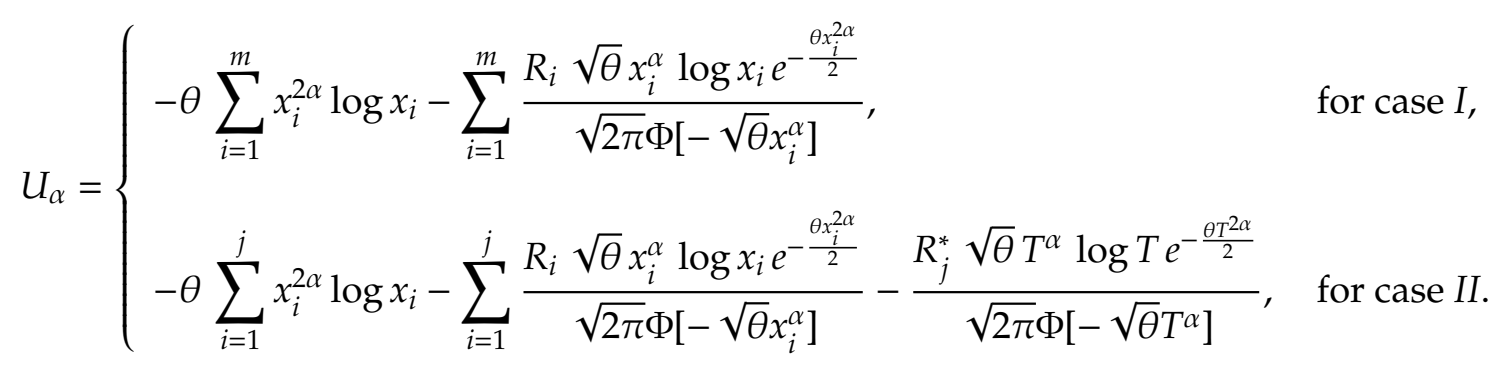


and

$$
U_{\theta}= \begin{cases}-\frac{1}{2} \sum_{i=1}^{m} x_{i}^{2 \alpha}-\sum_{i=1}^{m} \frac{R_{i} x_{i}^{\alpha} e^{-\frac{\theta x_{i}^{2 \alpha}}{2}}}{2 \sqrt{2 \pi \theta} \Phi\left[-\sqrt{\theta} x_{i}^{\alpha}\right]^{\prime}}, & \text { for case } I, \\ -\frac{1}{2} \sum_{i=1}^{j} x_{i}^{2 \alpha}-\sum_{i=1}^{j} \frac{R_{i} x_{i}^{\alpha} e^{-\frac{\theta x_{i}^{2 \alpha}}{2}}}{2 \sqrt{2 \pi \theta} \Phi\left[-\sqrt{\theta} x_{i}^{\alpha}\right]}-\frac{R_{j}^{*} T^{\alpha} e^{-\frac{\theta T^{2 \alpha}}{2}}}{2 \sqrt{2 \pi \theta} \Phi\left[-\sqrt{\theta} T^{\alpha}\right]}, & \text { for case II. }\end{cases}
$$

These likelihood equations are nonlinear in nature and it is relatively difficult to compute analytic expressions for respective MLEs $\hat{\alpha}$ and $\hat{\theta}$ of parameters $\alpha$ and $\theta$. However, these estimates can be computed numerically. Here we use an expectationmaximization (EM) algorithm for this purpose which is discussed next.

\subsection{EM Algorithm}

In this section, we apply an EM algorithm to compute MLEs of unknown parameters of the $G H N(\alpha, \theta)$ distribution. Dempster et al. (1977) initially proposed this algorithm in the literature as an iterative technique to compute maximum likelihood estimates of parameters in presence of censored observations. At each step of this algorithm two steps are implemented, namely the expectation-step and the maximization-step.

Suppose for the first case we have $X=\left(X_{(1)}, X_{(2)}, \cdots, X_{(m)}\right)$ as the observed data and $Y=\left(Y_{1}, Y_{2}, \cdots, Y_{m}\right)$ as the censored data where $Z_{g}$ denotes a $1 \times R_{g}$ vector with $Z_{g}=$ $\left(Y_{g 1}, Y_{g 2}, \cdots, Y_{g R}\right), g=1,2, \cdots, m$. So the complete data set is obtained as $Z=(X, Y)$ for the case $I$. Similarly for the other case let $X=\left(X_{(1)}, X_{(2)}, \cdots, X_{(j)}\right)$ be the observed data and $Y=\left(Y_{1}, Y_{2}, \cdots, Y_{j}\right)$ be the censored data with $Y_{s}$ being a $1 \times R_{S}$ vector such that $Y_{s}=\left(Y_{s 1}, Y_{s 2}, \cdots, Y_{s R}\right), s=1,2, \cdots, j$ and $Y^{\prime}=\left(Y_{1}^{\prime}, Y_{2}^{\prime}, \cdots, Y_{R_{j}^{*}}^{\prime}\right)$. So the complete data set for the case $I I$ is obtained as $Z=\left(X, Y, Y^{\prime}\right)$. Following Alma (2016), the likelihood function of $\alpha$ and $\theta$ is now given by

$$
\log L(\alpha, \theta \mid x)= \begin{cases}M, & \text { for case } I \\ M+N, & \text { for case } I I\end{cases}
$$


where

$$
\begin{aligned}
M & =\frac{n}{2} \log \frac{2}{\pi}+n \log \alpha+\frac{n}{2} \log \theta+(\alpha-1)\left(\sum_{i=1}^{d} \log x_{i: n}+\sum_{i=1}^{d} \sum_{k=1}^{R_{i}} \log y_{i k}\right) \\
& -\frac{\theta}{2}\left(\sum_{i=1}^{d} x_{i: n}^{2 \alpha}+\sum_{i=1}^{d} \sum_{k=1}^{R_{i}} y_{i k}^{2 \alpha}\right)
\end{aligned}
$$

and

$$
N=(\alpha-1) \sum_{l=1}^{R_{j}^{*}} \log y_{l}^{\prime}-\frac{\theta}{2} \sum_{l=1}^{R_{j}^{*}} y_{l}^{\prime 2 \alpha}
$$

After applying the expectation-step, $M$ and $N$ are re-expressed as

$$
\begin{aligned}
M & =\frac{n}{2} \log \frac{2}{\pi}+n \log \alpha+\frac{n}{2} \log \theta+(\alpha-1) \sum_{i=1}^{d} \log x_{i: n}-\frac{\theta}{2} \sum_{i=1}^{d} x_{i: n}^{2 \alpha} \\
& +(\alpha-1) \sum_{i=1}^{d} \sum_{k=1}^{R_{i}} E\left[\log Y_{i k} \mid Y_{i k}>x_{i}\right]-\frac{\theta}{2} \sum_{i=1}^{d} \sum_{k=1}^{R_{i}} E\left[Y_{i k}^{2 \alpha} \mid Y_{i k}>x_{i}\right]
\end{aligned}
$$

and

$$
N=(\alpha-1) \sum_{l=1}^{R_{j}^{*}} E\left[\log Y_{l}^{\prime} \mid Y_{l}^{\prime}>T\right]-\frac{\theta}{2} \sum_{l=1}^{R_{j}^{*}} E\left[Y_{l}^{\prime 2 \alpha} \mid Y_{l}^{\prime}>T\right]
$$

where required expectations are computed as

$$
\begin{aligned}
E\left(\log Y_{i k} \mid Y_{i k}>x_{i}\right) & =\frac{\alpha \sqrt{\frac{2 \theta}{\pi}}}{1-F_{X}\left(x_{i} ; \alpha, \theta\right)} \int_{x_{i}}^{\infty} \log y y^{\alpha-1} \exp \left\{-\frac{\theta}{2} y^{2 \alpha}\right\} d y \\
& =\frac{1}{\alpha \sqrt{2 \pi} \Phi\left[-\sqrt{\theta} x_{i}^{\alpha}\right]} \int_{\sqrt{\theta} x_{i}^{\alpha}}^{\infty} \log \left(\frac{t}{\sqrt{\theta}}\right) e^{-\frac{t^{2}}{2}} d t \\
& =A\left(x_{i} ; \alpha, \theta\right),
\end{aligned}
$$




$$
\begin{aligned}
E\left(Y_{i k}^{2 \alpha} \mid Y_{i}>x_{i}\right) & =\frac{\alpha \sqrt{\frac{2 \theta}{\pi}}}{1-F_{X}\left(x_{i} ; \alpha, \theta\right)} \int_{x_{i}}^{\infty} y^{2 \alpha} y^{\alpha-1} \exp \left\{-\frac{\theta}{2} y^{2 \alpha}\right\} d y \\
& =\frac{1}{\theta \sqrt{2 \pi} \Phi\left[-\sqrt{\theta} x_{i}^{\alpha}\right]} \int_{\sqrt{\theta} x_{i}^{\alpha}}^{\infty} t^{2} e^{-\frac{t^{2}}{2}} d t \\
& =B\left(x_{i} ; \alpha, \theta\right), \\
E\left(\log Y_{l}^{\prime} \mid Y_{l}^{\prime}>T\right) & =\frac{\alpha \sqrt{\frac{2 \theta}{\pi}}}{1-F_{X}(T ; \alpha, \theta)} \int_{T}^{\infty} \log y y^{\alpha-1} \exp \left\{-\frac{\theta}{2} y^{2 \alpha}\right\} d y \\
& =\frac{1}{\alpha \sqrt{2 \pi} \Phi\left[-\sqrt{\theta} T^{\alpha}\right]} \int_{\sqrt{\theta} T^{\alpha}}^{\infty} \log \left(\frac{t}{\sqrt{\theta}}\right) e^{-\frac{t^{2}}{2}} d t \\
& =C(T ; \alpha, \theta),
\end{aligned}
$$

and

$$
\begin{aligned}
E\left(Y_{l}^{\prime 2 \alpha} \mid Y_{l}^{\prime}>T\right) & =\frac{\alpha \sqrt{\frac{2 \theta}{\pi}}}{1-F_{X}(T ; \alpha, \theta)} \int_{T}^{\infty} y^{2 \alpha} y^{\alpha-1} \exp \left\{-\frac{\theta}{2} y^{2 \alpha}\right\} d y \\
& =\frac{1}{\theta \sqrt{2 \pi} \Phi\left[-\sqrt{\theta} T^{\alpha}\right]} \int_{\sqrt{\theta} T^{\alpha}}^{\infty} t^{2} e^{-\frac{t^{2}}{2}} d t \\
& =D(T ; \alpha, \theta) .
\end{aligned}
$$

Thus we are able to rewrite $M$ and $N$ as

$$
\begin{aligned}
M & =\frac{n}{2} \log \frac{2}{\pi}+n \log \alpha+\frac{n}{2} \log \theta+(\alpha-1) \sum_{i=1}^{d} \log x_{i}-\frac{\theta}{2} \sum_{i=1}^{d} x_{i}^{2 \alpha} \\
& +(\alpha-1) \sum_{i=1}^{d} R_{i} A\left(x_{i} ; \alpha, \theta\right)-\frac{\theta}{2} \sum_{i=1}^{d} R_{i} B\left(x_{i} ; \alpha, \theta\right),
\end{aligned}
$$

and

$$
N=R_{j}^{*}\left[(\alpha-1) C(T ; \alpha, \theta)-\frac{\theta}{2} D(T ; \alpha, \theta)\right]
$$

In the maximization-step, we maximize $M$ and $N$ with respect to $\alpha$ and $\theta$. As an 
example of case $I I$, given the $j$ th stage estimate of both of the parameters, in $(j+1)$ th iteration we solve the following nonlinear equation

$$
\frac{n}{\alpha}+\sum_{i=1}^{d} \log x_{i}-\hat{\theta}(\alpha) \sum_{i=1}^{d} x_{i}^{2 \alpha} \log x_{i}+\frac{1}{2} \sum_{i=1}^{d} R_{i} A\left(x_{i} ; \alpha_{(j)}, \theta_{(j)}\right)+R_{j}^{*} C\left(T ; \alpha_{(j)}, \theta_{(j)}\right)=0
$$

and obtain the $(j+1)$ th updated estimate of parameter $\alpha$. Accordingly updated the estimate of $\theta$ is obtained as

$$
\hat{\theta}(\alpha)=-\frac{n}{\sum_{i=1}^{d}\left[R_{i} B\left(x_{i} ; \alpha_{(j)}, \theta_{(j)}\right)+x_{i}^{2 \alpha}\right]+\frac{R_{j}^{*}}{2} D\left(T ; \alpha_{(j)}, \theta_{(j)}\right)} .
$$

We repeat this iterative process till a desired accuracy is achieved. Proceeding similarly MLEs of $\alpha$ and $\theta$ can be computed for the case $I$ where $R_{j}^{*}=0$. In the next section, we discuss the observed Fisher information matrix.

\subsection{Fisher Information Matrix}

In this section, we discuss the observed Fisher information matrix which is useful in computing interval estimates of parameters $\alpha$ and $\theta$ of the generalized half-normal distribution. We compute this matrix using the Louis (1982) method, according to which

$$
I_{X}(\beta)=I_{Z}(\beta)-I_{Z \mid X}(\beta),
$$

where $I_{X}(\beta), I_{Z}(\beta)$ and $I_{Z \mid X}(\beta)$ respectively denote the observed, the complete and the missing information, also let $\beta=(\alpha, \theta)$. We further have

$$
I_{Z \mid X}(\beta)= \begin{cases}\sum_{i=1}^{m} I_{Z \mid X}^{(i)}(\beta), & \text { for case } I, \\ \sum_{i=1}^{j} I_{Z \mid X}^{(i)}(\beta)+R_{j}^{*} I_{Z \mid T}(\beta), & \text { for case } I I .\end{cases}
$$

Similarly,

$$
I_{X}(\beta)=-E\left[\frac{\partial^{2} L_{c}(Z ; \beta)}{\partial \beta^{2}}\right], \quad I_{Z \mid X}^{(i)}(\beta)=-E_{Y_{i} \mid x_{(i)}}\left[\frac{\partial^{2} \ln f_{Y_{i}}\left(y_{i} \mid x_{(i)}, \beta\right)}{\partial \beta^{2}}\right],
$$


and

$$
I_{Z \mid T}(\beta)=-E_{Y^{\prime} \mid T}\left[\frac{\partial^{2} \ln f_{Y^{\prime}}\left(y^{\prime} \mid T, \beta\right)}{\partial \beta^{2}}\right] .
$$

For the generalize half-normal distribution the complete information is of the form

$$
I_{Z}(\beta)=\left[\begin{array}{ll}
l_{11} & l_{12} \\
l_{21} & l_{22}
\end{array}\right] .
$$

For case $I I$, elements of the above matrix are computed as

$$
\begin{aligned}
& l_{11}=\frac{n+R_{j}^{*}}{\alpha^{2}}+\frac{2\left(n+R_{j}^{*}\right) \alpha \theta \sqrt{2 \theta}}{\sqrt{\pi}} \int_{0}^{\infty} x^{2 \alpha}(\log x)^{2} x^{\alpha-1} e^{-\frac{\theta x^{2 \alpha}}{2}} d x, \\
& l_{12}=l_{21}=\frac{\left(n+R_{j}^{*}\right) \alpha \sqrt{2 \theta}}{\sqrt{\pi}} \int_{0}^{\infty} x^{2 \alpha} \log x x^{\alpha-1} e^{-\frac{\theta x^{2 \alpha}}{2}} d x, \\
& l_{22}=\frac{n+R_{j}^{*}}{\theta^{2}} .
\end{aligned}
$$

The corresponding elements under the case $I$ are obtained by putting $R_{j}^{*}=0$. Also, if $I_{Z \mid X}^{(i)}(\beta)$ denote the information matrix of a single observation truncated at $x_{i}$ then we have

where

$$
I_{Z \mid X}^{(i)}(\beta)=\left[\begin{array}{ll}
p_{11}\left(x_{(i)} ; \alpha, \theta\right) & p_{12}\left(x_{(i)} ; \alpha, \theta\right) \\
p_{21}\left(x_{(i)} ; \alpha, \theta\right) & p_{22}\left(x_{(i)} ; \alpha, \theta\right)
\end{array}\right],
$$

$$
\begin{aligned}
p_{11}\left(x_{(i)} ; \alpha, \theta\right) & =\frac{1}{\alpha^{2}}+\frac{2 \sqrt{2 \theta}}{\alpha^{2} \sqrt{\pi}} h_{1}\left(x_{i} ; \alpha, \theta\right)-\frac{\sqrt{\theta}\left(\log x_{i}\right)^{2} x_{i}^{\alpha} e^{-\frac{\theta x_{i}^{2 \alpha}}{2}}}{\sqrt{2 \pi} \Phi\left[-\sqrt{\theta} x_{i}^{\alpha}\right]} \\
& +\frac{\sqrt{\theta}\left(\log x_{i}\right)^{2} \theta x_{i}^{3 \alpha} e^{-\frac{\theta x_{i}^{2 \alpha}}{2}}}{\sqrt{2 \pi} \Phi\left[-\sqrt{\theta} x_{i}^{\alpha}\right]}-\frac{\theta\left(\log x_{i}\right)^{2}\left(x_{i}^{\alpha}\right)^{2} e^{-\theta x_{i}^{2 \alpha}}}{2 \pi\left(\Phi\left[-\sqrt{\theta} x_{i}^{\alpha}\right]\right)^{2}}, \\
p_{12}\left(x_{(i)} ; \alpha, \theta\right) & =\frac{\sqrt{2 \theta}}{\alpha \sqrt{\pi}} h_{2}\left(x_{i} ; \alpha, \theta\right)+\frac{\sqrt{\theta} \log x_{i} x_{i}^{2 \alpha} e^{-\frac{\theta x_{i}^{2 \alpha}}{2}}}{2 \sqrt{2 \pi} \Phi\left[-\sqrt{\theta} x_{i}^{\alpha}\right]} \\
& -\frac{\log x_{i} x_{i}^{\alpha} e^{-\theta x_{i}^{2 \alpha}}}{4 \pi\left(\Phi\left[-\sqrt{\theta} x_{i}^{\alpha}\right]\right)^{2}}=p_{21}\left(x_{(i)} ; \alpha, \theta\right),
\end{aligned}
$$


and

$$
p_{22}\left(x_{(i)} ; \alpha, \theta\right)=\frac{1}{2 \theta^{2}}+\frac{x_{i}^{2 \alpha} e^{-\frac{\theta x_{i}^{2 \alpha}}{2}}}{4 \sqrt{2 \pi \theta} \Phi\left[-\sqrt{\theta} x_{i}^{\alpha}\right]}+\frac{e^{-\frac{\theta x_{i}^{2 \alpha}}{2}}}{4 \sqrt{2 \pi} \theta^{\frac{3}{2}} \Phi\left[-\sqrt{\theta} x_{i}^{\alpha}\right]}-\frac{x_{i}^{\alpha} e^{-\theta x_{i}^{2 \alpha}}}{8 \pi \theta\left(\Phi\left[-\sqrt{\theta} x_{i}^{\alpha}\right]\right)^{2}} .
$$

The functions $h_{1}$ and $h_{2}$ are given by

$$
h_{1}\left(x_{i} ; \alpha, \theta\right)=\int_{0}^{\infty} u^{2}(\log u)^{2} e^{-\frac{\theta u^{2}}{2}} d u,
$$

and

$$
h_{2}\left(x_{i} ; \alpha, \theta\right)=\int_{0}^{\infty} u^{2} \log u e^{-\frac{\theta u^{2}}{2}} d u
$$

Similarly, if $I_{Z \mid T}(\beta)$ is the information in a single observation truncated at $T$, then we have

$$
I_{Z \mid T}(\beta)=\left[\begin{array}{ll}
q_{11}(T ; \alpha, \theta) & q_{12}(T ; \alpha, \theta) \\
q_{21}(T ; \alpha, \theta) & q_{22}(T ; \alpha, \theta)
\end{array}\right]
$$

where $q_{i j}(T ; \alpha, \theta) ; i, j=1,2$ is identical to respective $p_{i j}\left(x_{i} ; \alpha, \theta\right) ; i, j=1,2$ with $x_{i}$ being replaced by $T$. Thus the observed Fisher information matrix can be computed from Equation. (2.6) using above computations. The associated asymptotic variancecovariance matrix of MLEs of $\alpha$ and $\theta$ can be derived by inverting the observed Fisher information matrix. Subsequently, asymptotic intervals can be constructed using this matrix.

\section{Bayesian Estimation}

In this section, we obtain Bayes estimators of unknown parameters of the $G H N(\alpha, \theta)$ distribution under Type II progressive hybrid censoring when the loss function is squared error given by

$$
L_{1}(\mu, \delta)=(\delta-\mu)^{2} .
$$

Let $X_{1}, X_{2}, \ldots, X_{d}$ be a Type II progressive hybrid censored sample from a $G H N(\alpha, \theta)$ distribution. We assume that $\alpha$ and $\theta$ are independently a priori distributed as gamma $G(a, b)$ and $G(p, q)$ distributions respectively with joint prior distribution being

$$
\pi(\alpha, \theta) \propto \alpha^{a-1} e^{-b \alpha} \theta^{p-1} e^{-q \theta}, \quad \alpha>0, \theta>0, a>0, b>0, p>0, q>0,
$$


and the posterior distribution is obtained as

$$
\pi(\alpha, \theta \mid x)=k^{-1} \alpha^{d+a-1} \theta^{\frac{d}{2}+p-1} e^{-q \theta} e^{U(\alpha, \theta)} e^{-\alpha\left(b-\sum_{i=1}^{d} \log x_{i}\right)},
$$

where

$$
k=\int_{0}^{\infty} \int_{0}^{\infty} \alpha^{d+a-1} \theta^{\frac{d}{2}+p-1} e^{-q \theta} e^{U(\alpha, \theta)} e^{-\alpha\left(b-\sum_{i=1}^{d} \log x_{i}\right)} d \alpha d \theta .
$$

Now the Bayes estimator of $g(\alpha, \theta)$ under the squared error loss function is given by

$$
\hat{g}_{B}(\alpha, \theta)=E_{\alpha, \theta \mid x}(g(\alpha, \theta))=\frac{\int_{0}^{\infty} \int_{0}^{\infty} g(\alpha, \theta) e^{l(\alpha, \theta \mid x)+\rho(\alpha, \theta)} d \alpha d \theta}{\int_{0}^{\infty} \int_{0}^{\infty} e^{l(\alpha, \theta \mid x)+\rho(\alpha, \theta)} d \alpha d \theta},
$$

where $l(\alpha, \theta \mid x)$ is the log-likelihood function and $\rho(\alpha, \theta)=\log \pi(\alpha, \theta)$. In general, this Bayes estimator cannot be obtained in explicit form as the corresponding posterior distribution is analytically intractable. However approximate Bayes estimator can be computed using the Tierney and Kadane (1986) method which is discussed in the next section.

\subsection{Tierney and Kadane Method}

Here we obtain an explicit expression for the Bayes estimator of $g(\alpha, \theta)$. We first define functions

$$
\delta(\alpha, \theta)=\frac{l(\alpha, \theta \mid x)+\rho(\alpha, \theta)}{n}, \quad \text { and } \quad \delta_{\beta}^{*}(\alpha, \theta)=\delta(\alpha, \theta)+\frac{\log g(\alpha, \theta)}{n} .
$$

Let $\left(\hat{\alpha}_{\delta}, \hat{\theta}_{\delta}\right)$ and $\left(\hat{\alpha}_{\delta^{*}}, \hat{\theta}_{\delta^{*}}\right)$ denote values of $(\alpha, \theta)$ which respectively maximize functions $\delta(\alpha, \theta)$ and $\delta_{\beta}^{*}(\alpha, \theta)$. Then using the TK method estimator, (B.4) is approximated as

$$
I(x)=\sqrt{\frac{\left|\Sigma_{\beta}^{*}\right|}{|\Sigma|}} \exp \left[n\left\{\delta_{\beta}^{*}\left(\hat{\alpha}_{\delta^{*}}, \hat{\theta}_{\delta^{*}}\right)-\delta\left(\hat{\alpha}_{\delta}, \hat{\theta}_{\delta}\right)\right\}\right]
$$

where $|\Sigma|$ and $\left|\Sigma_{\beta}^{*}\right|$ are the negatives of inverse Hessians of $\delta(\alpha, \theta)$ and $\delta_{\beta}^{*}(\alpha, \theta)$ respectively computed at $\left(\hat{\alpha}_{\delta}, \hat{\theta}_{\delta}\right)$ and $\left(\hat{\alpha}_{\delta^{*}}, \hat{\theta}_{\delta^{*}}\right)$. Further for the generalized half-normal 
distribution, we have

$$
\begin{aligned}
\delta(\alpha, \theta) & =\frac{1}{n}\left\{(d+a-1) \log \alpha-\alpha\left(b-\sum_{i=1}^{d} \log x_{i}\right)+\left(\frac{d}{2}+p-1\right) \log \theta-\sum_{i=1}^{d} \log x_{i}\right. \\
& \left.-\theta\left(q+\frac{1}{2} \sum_{i=1}^{d} x_{i}^{2 \alpha}\right)+\sum_{i=1}^{d} R_{i} \log \Phi\left[-\sqrt{\theta} x_{i}^{\alpha}\right]+R_{j}^{*} \log \Phi\left[-\sqrt{\theta} T^{\alpha}\right]\right\},
\end{aligned}
$$

and then we obtain $\left(\hat{\alpha}_{\delta}, \hat{\theta}_{\delta}\right)$ by simultaneously solving the following nonlinear equations

$$
\begin{aligned}
\frac{\partial \delta}{\partial \alpha} & =\frac{(d+a-1)}{n \alpha}-\frac{1}{n}\left\{b-\sum_{i=1}^{d} \log x_{i}+\theta \sum_{i=1}^{d} x_{i}^{2 \alpha} \log x_{i}+\sum_{i=1}^{d} \frac{R_{i} \sqrt{\theta} x_{i}^{\alpha} \log x_{i} e^{-\frac{\theta x_{i}^{2 \alpha}}{2}}}{\sqrt{2 \pi} \Phi\left[-\sqrt{\theta} x_{i}^{\alpha}\right]}\right. \\
& \left.+\frac{R_{j}^{*} \sqrt{\theta} x_{i}^{\alpha} \log T e^{-\frac{\theta T^{2 \alpha}}{2}}}{\sqrt{2 \pi} \Phi\left[-\sqrt{\theta} T^{\alpha}\right]}\right\}=0, \\
\frac{\partial \delta}{\partial \theta} & =\frac{\left(\frac{d}{2}+p-1\right)}{n \theta}-\frac{1}{n}\left\{q+\frac{1}{2} \sum_{i=1}^{d} x_{i}^{2 \alpha}+\sum_{i=1}^{d} \frac{R_{i} x_{i}^{\alpha} e^{-\frac{\theta x_{i}^{2 \alpha}}{2}}}{2 \sqrt{2 \pi \theta} \Phi\left[-\sqrt{\theta} x_{i}^{\alpha}\right]}+\frac{R_{j}^{*} T^{\alpha} e^{-\frac{\theta T^{2 \alpha}}{2}}}{2 \sqrt{2 \pi \theta} \Phi\left[-\sqrt{\theta} T^{\alpha}\right]}\right\}=0 .
\end{aligned}
$$

Using the following second order partial derivatives of $\delta(\alpha, \theta)$

$$
\begin{aligned}
\frac{\partial^{2} \delta}{\partial \alpha^{2}} & =-\frac{(d+a-1)}{n \alpha^{2}}-\frac{1}{n}\left\{2 \theta \sum_{i=1}^{d} x_{i}^{2 \alpha}\left(\log x_{i}\right)^{2}+\sum_{i=1}^{d} \frac{R_{i} \sqrt{\theta}\left(\log x_{i}\right)^{2} x_{i}^{\alpha} e^{-\frac{\theta x_{i}^{2 \alpha}}{2}}}{\sqrt{2 \pi} \Phi\left[-\sqrt{\theta} x_{i}^{\alpha}\right]}\right. \\
& -\sum_{i=1}^{d} \frac{R_{i} \theta^{\frac{3}{2}}\left(\log x_{i}\right)^{2} x_{i}^{3 \alpha} e^{-\frac{\theta x_{i}^{2 \alpha}}{2}}}{\sqrt{2 \pi} \Phi\left[-\sqrt{\theta} x_{i}^{\alpha}\right]}+\sum_{i=1}^{d} \frac{R_{i} \theta\left(\log x_{i}\right)^{2} x_{i}^{2 \alpha} e^{-\theta x_{i}^{2 \alpha}}}{2 \pi\left(\Phi\left[-\sqrt{\theta} x_{i}^{\alpha}\right]\right)^{2}}+\frac{R_{j}^{*} \sqrt{\theta}(\log T)^{2} T^{\alpha} e^{-\frac{\theta T^{2 \alpha}}{2}}}{\sqrt{2 \pi} \Phi\left[-\sqrt{\theta} T^{\alpha}\right]} \\
& \left.-\frac{R_{j}^{*} \theta^{\frac{3}{2}}(\log T)^{2} T^{3 \alpha} e^{-\frac{\theta T^{2 \alpha}}{2}}}{\sqrt{2 \pi} \Phi\left[-\sqrt{\theta} T^{\alpha}\right]}+\frac{R_{j}^{*} \theta(\log T)^{2} T^{2 \alpha} e^{-\theta T^{2 \alpha}}}{2 \pi\left(\Phi\left[-\sqrt{\theta} T^{\alpha}\right]\right)^{2}}\right\},
\end{aligned}
$$




$$
\begin{aligned}
\frac{\partial^{2} \delta}{\partial \theta \partial \alpha}=\frac{\partial^{2} \delta}{\partial \alpha \partial \theta}= & -\frac{1}{n}\left\{\sum_{i=1}^{d} x_{i}^{2 \alpha} \log x_{i}+\sum_{i=1}^{d} \frac{R_{i} \log x_{i} x_{i}^{\alpha} e^{-\frac{\theta x_{i}^{2 \alpha}}{2}}}{2 \sqrt{2 \theta \pi} \Phi\left[-\sqrt{\theta} x_{i}^{\alpha}\right]}-\sum_{i=1}^{d} \frac{R_{i} \sqrt{\theta} \log x_{i} x_{i}^{3 \alpha} e^{-\frac{\theta x_{i}^{2 \alpha}}{2}}}{2 \sqrt{2 \pi} \Phi\left[-\sqrt{\theta} x_{i}^{\alpha}\right]}\right. \\
& +\sum_{i=1}^{d} \frac{R_{i} \log x_{i} x_{i}^{2 \alpha} e^{-\theta x_{i}^{2 \alpha}}}{4 \pi\left(\Phi\left[-\sqrt{\theta} x_{i}^{\alpha}\right]\right)^{2}}+\frac{R_{j}^{*} \log T T^{\alpha} e^{-\frac{\theta T^{2 \alpha}}{2}}}{2 \sqrt{2 \pi \theta} \Phi\left[-\sqrt{\theta} T^{\alpha}\right]}-\frac{R_{j}^{*} \sqrt{\theta} \log T T^{3 \alpha} e^{-\frac{\theta T^{2 \alpha}}{2}}}{2 \sqrt{2 \pi} \Phi\left[-\sqrt{\theta} T^{\alpha}\right]} \\
& \left.+\frac{R_{j}^{*} \log T T^{2 \alpha} e^{-\theta T^{2 \alpha}}}{4 \pi\left(\Phi\left[-\sqrt{\theta} T^{\alpha}\right]\right)^{2}}\right\}, \\
\frac{\partial^{2} \delta}{\partial \theta^{2}} & =-\frac{\left(\frac{d}{2}+p-1\right)}{n \theta^{2}}+\frac{1}{n}\left\{\sum_{i=1}^{d} \frac{R_{i} x_{i}^{\alpha} e^{-\frac{\theta x_{i}^{2 \alpha}}{2}}}{4 \sqrt{2 \pi} \theta^{\frac{3}{2}} \Phi\left[-\sqrt{\theta} x_{i}^{\alpha}\right]}+\sum_{i=1}^{d} \frac{R_{i} x_{i}^{3 \alpha} e^{-\frac{\theta x_{i}^{2 \alpha}}{2}}}{4 \sqrt{\theta} \sqrt{2 \pi} \Phi\left[-\sqrt{\theta} x_{i}^{\alpha}\right]}\right. \\
& -\sum_{i=1}^{d} \frac{R_{i} x_{i}^{2 \alpha} e^{-\theta x_{i}^{2 \alpha}}}{8 \pi \theta\left(\Phi\left[-\sqrt{\theta} x_{i}^{\alpha}\right]\right)^{2}}+\frac{R_{j}^{*} T^{-\frac{\theta T^{2 \alpha}}{2}} e^{-\frac{\theta T^{2 \alpha}}{2}}}{4 \sqrt{2 \pi} \theta^{\frac{3}{2}} \Phi\left[-\sqrt{\theta} T^{\alpha}\right]}+\frac{R_{j}^{*} T^{2 \alpha} e^{-\theta T^{2 \alpha}}}{4 \sqrt{2 \pi \theta} \Phi\left[-\sqrt{\theta} T^{\alpha}\right]} \\
& -\frac{8 \pi \theta\left(\Phi\left[-\sqrt{\theta} T^{\alpha}\right]\right)^{2}}{8 \pi},
\end{aligned}
$$

we compute $|\Sigma|$ as

$$
|\Sigma|=\left[\frac{\partial^{2} \delta}{\partial \alpha^{2}} * \frac{\partial^{2} \delta}{\partial \theta^{2}}-\frac{\partial^{2} \delta}{\partial \alpha \partial \theta} * \frac{\partial^{2} \delta}{\partial \theta \partial \alpha}\right]^{-1} .
$$

We consider $g(\alpha, \theta)=\alpha$ for estimating the parameter $\alpha$. The quantity $\left(\hat{\alpha}_{\delta^{*}}, \hat{\theta}_{\delta^{*}}\right)$ is then computed from the function $\delta_{\alpha}^{*}(\alpha, \theta)$, where

$$
\delta_{\alpha}^{*}(\alpha, \theta)=\delta(\alpha, \theta)+\frac{1}{n} \log \alpha,
$$

and it is obtained as the solution of the following non-linear equations

$$
\frac{\partial \delta_{\alpha}^{*}}{\partial \alpha}=\frac{\partial \delta}{\partial \alpha}+\frac{1}{n \alpha}=0, \quad \frac{\partial \delta_{\alpha}^{*}}{\partial \theta}=\frac{\partial \delta}{\partial \theta}=0 .
$$

Again using the following second order partial derivatives of $\delta_{\alpha}^{*}(\alpha, \theta)$

$$
\frac{\partial^{2} \delta_{\alpha}^{*}}{\partial \alpha^{2}}=\frac{\partial^{2} \delta}{\partial \alpha^{2}}-\frac{1}{n \alpha^{2}}, \quad \frac{\partial^{2} \delta_{\alpha}^{*}}{\partial \theta \partial \alpha}=\frac{\partial^{2} \delta_{\alpha}^{*}}{\partial \alpha \partial \theta}=\frac{\partial^{2} \delta}{\partial \theta \partial \alpha}, \quad \frac{\partial^{2} \delta_{\alpha}^{*}}{\partial \theta^{2}}=\frac{\partial^{2} \delta}{\partial \theta^{2}} .
$$


We are able to compute $\left|\Sigma_{\alpha}^{*}\right|$ as

$$
\left|\Sigma_{\alpha}^{*}\right|=\left[\frac{\partial^{2} \delta_{\alpha}^{*}}{\partial \alpha^{2}} * \frac{\partial^{2} \delta_{\alpha}^{*}}{\partial \theta^{2}}-\frac{\partial^{2} \delta_{\alpha}^{*}}{\partial \alpha \partial \theta} * \frac{\partial^{2} \delta_{\alpha}^{*}}{\partial \theta \partial \alpha}\right]^{-1}
$$

The Bayes estimator of $\alpha$ is now given by

$$
\tilde{\alpha}_{T K}=\sqrt{\frac{\left|\Sigma_{\alpha}^{*}\right|}{|\Sigma|}} \exp \left[n\left\{\delta_{\alpha}^{*}\left(\hat{\alpha}_{\delta^{*}}, \hat{\theta}_{\delta^{*}}\right)-\delta\left(\hat{\alpha}_{\delta}, \hat{\theta}_{\delta}\right)\right\}\right] .
$$

Similarly, for estimating $\theta$ we consider $g(\alpha, \theta)=\theta$ and define

$$
\delta_{\theta}^{*}(\alpha, \theta)=\delta(\alpha, \theta)+\frac{1}{n} \log \theta
$$

We compute $\left(\hat{\alpha}_{\delta^{*}}, \hat{\theta}_{\delta^{*}}\right)$ from the following equations

$$
\frac{\partial \delta_{\theta}^{*}}{\partial \alpha}=\frac{\partial \delta}{\partial \alpha}=0, \quad \frac{\partial \delta_{\theta}^{*}}{\partial \theta}=\frac{\partial \delta}{\partial \theta}+\frac{1}{n \theta}=0,
$$

and using the second order partial derivatives of $\delta_{\theta}^{*}(\alpha, \theta)$

$$
\frac{\partial^{2} \delta_{\theta}^{*}}{\partial \alpha^{2}}=\frac{\partial^{2} \delta}{\partial \alpha^{2}}, \quad \frac{\partial^{2} \delta_{\theta}^{*}}{\partial \theta \partial \alpha}=\frac{\partial^{2} \delta_{\theta}^{*}}{\partial \alpha \partial \theta}=\frac{\partial^{2} \delta}{\partial \theta \partial \alpha}, \quad \frac{\partial^{2} \delta_{\theta}^{*}}{\partial \theta^{2}}=\frac{\partial^{2} \delta}{\partial \theta^{2}}-\frac{1}{n \theta^{2}} .
$$

We compute

$$
\left|\Sigma_{\theta}^{*}\right|=\left[\frac{\partial^{2} \delta_{\theta}^{*}}{\partial \alpha^{2}} * \frac{\partial^{2} \delta_{\theta}^{*}}{\partial \theta^{2}}-\frac{\partial^{2} \delta_{\theta}^{*}}{\partial \alpha \partial \theta} * \frac{\partial^{2} \delta_{\theta}^{*}}{\partial \theta \partial \alpha}\right]^{-1} .
$$

The Bayes estimator of $\theta$ is finally obtained as

$$
\tilde{\theta}_{T K}=\sqrt{\frac{\left|\Sigma_{\theta}^{*}\right|}{|\Sigma|}} \exp \left[n\left\{\delta_{\theta}^{*}\left(\hat{\alpha}_{\delta^{*}}, \hat{\theta}_{\delta^{*}}\right)-\delta\left(\hat{\alpha}_{\delta}, \hat{\theta}_{\delta}\right)\right\}\right] .
$$

The Bayes estimators of unknown parameters can easily be computed from the TK method, however, this procedure is not suitable for the interval estimation. We now propose an importance sampling procedure which can be used to compute the Bayes estimates of the parameters and corresponding credible intervals. 


\subsection{Importance Sampling}

We propose an importance sampling scheme for computing Bayes estimates of the unknown parameters $\alpha$ and $\theta$ under the square error loss function when data are Type II progressive hybrid censored. We also obtain highest posterior density (HPD) intervals of these parameters. The posterior distribution of $\alpha$ and $\theta$ given the data is of the following form

$$
\pi(\alpha, \theta \mid x) \propto G_{\alpha}\left(d+a, b-\sum_{i=1}^{d} \log x_{i}\right) G_{\theta \mid \alpha}\left(\frac{d}{2}+p, q+\frac{1}{2} \sum_{i=1}^{d} x_{i}^{2 \alpha}\right) h(\alpha, \theta),
$$

where $h(\alpha, \theta)=V(\alpha, \theta)\left[q+\frac{1}{2} \sum_{i=1}^{d} x_{i: n}^{2 \alpha}\right]^{-\frac{d}{2}-p}$, and

$$
V(\alpha, \theta)= \begin{cases}e^{\sum_{i=1}^{m} R_{i} \log \Phi\left[-\sqrt{\theta} x_{i}^{\alpha}\right]}, & \text { for case } I, \\ e^{\sum_{i=1}^{j} R_{i} \log \Phi\left[-\sqrt{\theta} x_{i}^{\alpha}\right]+R_{j}^{*} \log \Phi\left[-\sqrt{\theta} T^{\alpha}\right]}, & \text { for case } I I .\end{cases}
$$

We generate posterior samples using the following steps.

Step 1 Generate $\alpha_{1} \sim G_{\alpha}(.,$.$) .$

Step 2 Generate $\theta_{1} \sim G_{\theta \mid \alpha}(.,$.$) .$

Step 3 Repeat the above two steps $s$ times to generate $\left(\alpha_{1}, \theta_{1}\right),\left(\alpha_{2}, \theta_{2}\right), \ldots,\left(\alpha_{s}, \theta_{s}\right)$.

The Bayes estimate of $g(\alpha, \theta)$ is computed as

$$
\tilde{g}_{I S}(\alpha, \theta)=\frac{\sum_{i=1}^{s} g\left(\alpha_{i}, \theta_{i}\right) h\left(\alpha_{i}, \theta_{i}\right)}{\sum_{i=1}^{s} h\left(\alpha_{i}, \theta_{i}\right)} .
$$

The $100(1-\tau) \%$ HPD interval of $\alpha$ and $\theta$ can be obtained from the method of Chen and Shao (1999). We have discussed the proposed intervals in the simulation study and real data analysis sections. 


\section{Prediction of Censored Observations}

\subsection{Point Prediction}

Here we obtain prediction estimates and prediction intervals of censored observations based on the observed Type II progressive hybrid censored data. Let $x=\left(x_{1}, x_{2}, \ldots, x_{d}\right)$ denote a Type II progressive hybrid censored sample from the generalized half-normal distribution using the censoring scheme $R=\left(R_{1}, R_{2}, \ldots, R_{d}\right)$. Let $y_{i}=\left(y_{i 1}, y_{i 2}, \ldots, y_{i R_{i}}\right)$ denotes lifetimes of observations censored at the $x_{i}$ th observation and we wish to predict the lifetime of $y=\left(y_{i k}, i=1,2, \cdots, d ; k=1,2, \cdots R_{i}\right)$. The conditional distribution of $y$ given the observed data is given by

$$
f(y \mid x, \alpha, \theta)=k\left(\begin{array}{c}
R_{i} \\
k
\end{array}\right) \frac{\left[F(y)-F\left(x_{i}\right)\right]^{k-1}[1-F(y)]^{R_{i}-k} f(y)}{\left[1-F\left(x_{i}\right)\right]^{R_{i}}}, \quad y>x_{i} .
$$

This is rewritten as

$$
f(y \mid x, \alpha, \theta)=k\left(\begin{array}{c}
R_{i} \\
k
\end{array}\right) \sum_{j=0}^{k-1}(-1)^{k-1-j}\left(\begin{array}{c}
k-1 \\
j
\end{array}\right)\left(1-F\left(x_{i}\right)\right)^{j-R_{i}}(1-F(y))^{R_{i}-1-j} f(y), \quad y>x_{i} .
$$

Below we obtain different predictors of censored observations.

\subsubsection{Maximum Likelihood Predictor}

Here we derive predictive estimates of censored observations using the maximum likelihood method. We write the predictive likelihood function (PLF) of $y$ and $(\alpha, \theta)$ as

$$
L(y, \alpha, \theta \mid x)=f(y \mid x, \alpha, \theta) f(x \mid \alpha, \theta)
$$

Suppose $\hat{Y}=u_{1}(x), \hat{\alpha}=u_{2}(x)$ and $\hat{\theta}=u_{3}(x)$ satisfy the following expression

$$
L\left(u_{1}(x), u_{2}(x), u_{3}(x) \mid x\right)=\sup _{(y, \alpha, \theta)} L(y, \alpha, \theta \mid x),
$$

then $u_{1}(x)$ is referred to as the maximum likelihood predictor (MLP) of the censored observation $y$ and $u_{2}(x)$ and $u_{3}(x)$ respectively denote predictive maximum likelihood estimators (PMLEs) of $\alpha$ and $\theta$. Now we express the predictive likelihood function of 
$y, \alpha$ and $\theta$ as

$$
\begin{aligned}
L(y, \alpha, \theta) & =\frac{1}{\sqrt{2 \pi}} k\left(\begin{array}{c}
R_{i} \\
k
\end{array}\right) \alpha^{(m+1)} \theta^{\frac{(m+1)}{2}} y^{\alpha-1}\left\{\prod_{i=1}^{m} x_{i}^{\alpha-1}\right\}\left[\Phi\left(-\sqrt{\theta} x_{i}^{\alpha}\right)-\Phi\left(-\sqrt{\theta} y^{\alpha}\right)\right]^{k-1} \\
& \times\left[\Phi\left(-\sqrt{\theta} y^{\alpha}\right)\right]^{R_{i}-k} \exp \left(-\frac{\theta}{2}\left\{y^{2 \alpha}+\sum_{i=1}^{m} x_{i}^{2 \alpha}\right\}\right) .
\end{aligned}
$$

The predictive log-likelihood function, after ignoring constant terms, is given by

$$
\begin{aligned}
\log L(y, \alpha, \theta) & \propto(m+1) \log \alpha+\frac{(m+1)}{2} \log \theta+(\alpha-1)\left(\log y+\sum_{i=1}^{m} \log x_{i}\right)-\frac{\theta}{2}\left(y^{2 \alpha}+\sum_{i=1}^{m} x_{i}^{2 \alpha}\right) \\
& +(k-1) \log \left(\Phi\left(-\sqrt{\theta} x_{i}^{\alpha}\right)-\Phi\left(-\sqrt{\theta} y^{\alpha}\right)\right)+\left(R_{i}-k\right) \log \left(\Phi\left(-\sqrt{\theta} y^{\alpha}\right)\right) .
\end{aligned}
$$

Subsequently, the predictive likelihood equations (PLEs) for $y, \alpha$ and $\theta$ are obtained as follows

$$
\begin{aligned}
\frac{\partial \log L(y, \alpha, \theta)}{\partial y} & =\frac{\alpha-1}{y}-\alpha \theta y^{2 \alpha-1}-\frac{\left(R_{i}-k\right) \alpha \sqrt{\theta} y^{\alpha-1} e^{\frac{-\theta y^{2 \alpha}}{2}}}{\sqrt{2 \pi} \Phi\left(-\sqrt{\theta} y^{\alpha}\right)} \\
& +\frac{(k-1) \alpha \sqrt{\theta} y^{\alpha-1} e^{\frac{-\theta y^{2 \alpha}}{2}}}{\sqrt{2 \pi}\left(\Phi\left(-\sqrt{\theta} x_{i}^{\alpha}\right)-\Phi\left(-\sqrt{\theta} y^{\alpha}\right)\right)}
\end{aligned}
$$

$$
\begin{aligned}
\frac{\partial \log L(y, \alpha, \theta)}{\partial \alpha} & =\frac{m+1}{\alpha}+\log y-\theta\left(y^{2 \alpha} \log y+\sum_{i=1}^{m} x_{i}^{2 \alpha} \log x_{i}\right)-\frac{\left(R_{i}-k\right) \sqrt{\theta} y^{\alpha} \log y e^{\frac{-\theta y^{2 \alpha}}{2}}}{\sqrt{2 \pi} \Phi\left(-\sqrt{\theta} y^{\alpha}\right)} \\
& +\sum_{i=1}^{m} \log x_{i}-\frac{(k-1) \sqrt{\theta}\left(y^{\alpha} \log y e^{\frac{-\theta y^{2 \alpha}}{2}}+x_{i}^{\alpha} \log x_{i} e^{\frac{-\theta x_{i}^{2 \alpha}}{2}}\right)}{\sqrt{2 \pi}\left(\Phi\left(-\sqrt{\theta} x_{i}^{\alpha}\right)-\Phi\left(-\sqrt{\theta} y^{\alpha}\right)\right)}
\end{aligned}
$$




$$
\begin{aligned}
\frac{\partial \log L(y, \alpha, \theta)}{\partial \theta} & =\frac{m+1}{2 \theta}-\frac{1}{2}\left(y^{2 \alpha}+\sum_{i=1}^{m} x_{i}^{2 \alpha}\right)-\frac{\left(R_{i}-k\right) y^{\alpha} e^{\frac{-\theta y^{2 \alpha}}{2}}}{2 \sqrt{2 \pi \theta} \Phi\left(-\sqrt{\theta} y^{\alpha}\right)} \\
& +\frac{(k-1)\left(y^{\alpha} e^{\frac{-\theta y^{2 \alpha}}{2}}-x_{i}^{\alpha} e^{\frac{-\theta x_{i}^{2 \alpha}}{2}}\right)}{2 \sqrt{2 \pi \theta}\left(\Phi\left(-\sqrt{\theta} x_{i}^{\alpha}\right)-\Phi\left(-\sqrt{\theta} y^{\alpha}\right)\right)} .
\end{aligned}
$$

Solving Equations (4.6), (4.7) and (4.8) simultaneously, the MLP $\hat{y}$ of the censored observation $y$ is obtained. Similarly, PMLE $(\hat{\alpha}, \hat{\theta})$ of $(\alpha, \theta)$ is obtained. In the next section, the best unbiased predictor (BUP) is discussed.

\subsubsection{Best Unbiased Predictor}

Here we obtain the best unbiased predictor (BUP) of the censored observation $y$. A statistic $\hat{y}$ that is used to predict $y$ is referred to as the BUP of $y$ if the prediction error $\hat{y}-y$ has zero mean and its prediction error variance $\operatorname{Var}(\hat{y}-y)$ is less than or equal to that of any other unbiased predictor of $y$. Note that conditional distribution of $y$ given the observed data $x=\left(x_{1}, x_{2}, \ldots, x_{d}\right)$ is the distribution of $y$ given the observed lifetime $x_{i}$. So BUP of $y$ is obtained as

$$
\begin{aligned}
\hat{y}_{\text {BUP }}=E(y \mid x) & =\int_{x_{i}}^{\infty} y f(y \mid x, \alpha, \theta) d y \\
& =\int_{x_{i}}^{\infty} y k\left(\begin{array}{c}
R_{i} \\
k
\end{array}\right) \frac{\left[F(y)-F\left(x_{i}\right)\right]^{k-1}[1-F(y)]^{R_{i}-k} f(y)}{\left[1-F\left(x_{i}\right)\right]^{R_{i}}} d y .
\end{aligned}
$$

After simplification, we rewrite the above expression as

$$
\hat{y}_{\text {BUP }}=\int_{x_{i}}^{\infty} k\left(\begin{array}{c}
R_{i} \\
k
\end{array}\right) y f(y)\left[1-F\left(x_{i}\right)\right]^{-1}\left[\frac{1-F(y)}{1-F\left(x_{i}\right)}\right]^{R_{i}-k}\left[1-\frac{1-F(y)}{1-F\left(x_{i}\right)}\right]^{k-1} d y .
$$

Consider the transformation $\left[\frac{1-F(y)}{1-F\left(x_{i}\right)}\right]=u$, then we have

$$
\hat{y}_{B U P}=\frac{1}{\operatorname{Beta}\left(R_{i}-k+1, k\right)} \int_{0}^{1}\left[-\frac{1}{\sqrt{\theta}} \Phi^{-1}\left(\frac{u F^{*}}{2}\right)\right]^{\frac{1}{\alpha}} u^{R_{i}-k}(1-u)^{k-1} d u,
$$


where $F^{*}=1-F\left(x_{i}\right)$. The parameters $\alpha$ and $\theta$ are unknown, so we need to estimate these parameters. We replace these parameters by their corresponding MLEs and then compute the desired BUP of $y$.

\subsubsection{Bayesian Predictor}

We obtain the Bayes predictor (BP) of censored observation $y$ against the squared error loss function. We observe that the posterior predictive density of $y$ given the data is of the form

$$
f^{*}(y \mid x)=\int_{0}^{\infty} \int_{0}^{\infty} f(y \mid \alpha, \theta, x) \pi(\alpha, \theta \mid x) d \alpha d \theta
$$

where $\pi(\alpha, \theta \mid x)$ denotes the joint posterior density of $\alpha$ and $\theta$. The Bayes predictor of $y$ is given by

$$
\begin{aligned}
\hat{y} & =\int_{x_{i}}^{\infty} y f^{*}(y \mid x) d y \\
& =\int_{0}^{\infty} \int_{0}^{\infty} \int_{x_{i}}^{\infty} y f(y \mid x, \alpha, \theta) \pi(\alpha, \theta \mid x) d y d \alpha d \theta \\
& =\int_{0}^{\infty} \int_{0}^{\infty} I\left(x_{i} \mid x, \alpha, \theta\right) \pi(\alpha, \theta \mid x) d \alpha d \theta,
\end{aligned}
$$

where

$$
\begin{aligned}
I\left(x_{i} \mid x, \alpha, \theta\right) & =\int_{x_{i}}^{\infty} y f(y) d y \\
& =k\left(\begin{array}{c}
R_{i} \\
k
\end{array}\right) \sum_{j=0}^{k-1}(-1)^{k-1-j}\left(\begin{array}{c}
k-1 \\
j
\end{array}\right) \int_{x_{i}}^{\infty} y\left(1-F\left(x_{i}\right)\right)^{j-R_{i}} f(y)(1-F(y))^{R_{i}-j-1} d y \\
& =k\left(\begin{array}{c}
R_{i} \\
k
\end{array}\right) \sum_{j=0}^{k-1}(-1)^{k-1-j}\left(\begin{array}{c}
k-1 \\
j
\end{array}\right)\left(2 \Phi\left(-\sqrt{\theta} x_{i}^{\alpha}\right)\right)^{\left(j-R_{i}\right)} \\
& \times \int_{0}^{2 \Phi\left(-\sqrt{\theta} x_{i}^{\alpha}\right)} z^{R_{i}-j-1}\left(-\frac{1}{\sqrt{\theta}} \Phi^{-1}\left(\frac{z}{2}\right)\right)^{\frac{1}{\alpha}} d z .
\end{aligned}
$$


The importance sampling technique is used to compute the corresponding predictive estimate $\hat{y}$. Let $\left\{\left(\alpha_{i}, \theta_{i}\right): i=1,2, \ldots, s\right\}$ be a sample from $\pi(\alpha, \theta \mid x)$, then we have

$$
\hat{y}=\frac{\sum_{i=1}^{s} I\left(\alpha_{i}, \theta_{i}\right) h\left(\alpha_{i}, \theta_{i}\right)}{\sum_{i=1}^{s} h\left(\alpha_{i}, \theta_{i}\right)} .
$$

Similarly, prediction estimates of censored observation that occur after the time point $T$ can be obtained. In this case, we replace $R_{i}$ and $x_{i}$ by $R_{j}^{*}$ and $T$ respectively and proceed similarly.

\subsection{Prediction Intervals}

Here we construct prediction intervals of $y=\left(y_{i k}, i=1,2, \cdots, d ; k=1,2, \cdots R_{i}\right)$ based on the observed type-II progressive hybrid data.

\subsubsection{Pivotal Method}

Consider the transformation $U=\left[\frac{1-F(y)}{1-F\left(x_{i}\right)}\right]$ and then we note that $U$ given $x$ follows a beta $\operatorname{Beta}\left(k, R_{i}-k+1\right)$ distribution. Furthermore, $U$ is a pivotal quantity and so prediction interval for $y$ can easily be computed using its distribution. Then the corresponding $100(1-\tau) \%$ interval is $\left(L_{1}(x), U_{1}(x)\right)$ where

$$
L_{1}(x)=\left[-\frac{1}{\sqrt{\theta}} \Phi^{-1}\left(\frac{F^{*}}{2}\left(1-B_{\frac{\tau}{2}}\right)\right)\right]^{\frac{1}{\alpha}}, \quad U_{1}(x)=\left[-\frac{1}{\sqrt{\theta}} \Phi^{-1}\left(\frac{F^{*}}{2}\left(1-B_{\left(1-\frac{\tau}{2}\right)}\right)\right)\right]^{\frac{1}{\alpha}}
$$

with $B_{\tau}$ denoting $100 \tau$ th percentile of $\operatorname{Beta}\left(k, R_{i}-k+1\right)$ distribution. Here $\alpha$ and $\theta$ can be replaced by their respective MLEs and accordingly prediction limits for $y$ can be obtained.

\subsubsection{Highest Conditional Density Method}

The distribution of $U$ given the observed data is a $\operatorname{Beta}\left(k, R_{i}-k+1\right)$ distribution with PDF

$$
g(u)=\frac{u^{k-1}(1-u)^{R_{i}-k}}{\operatorname{Beta}\left(k, R_{i}-k+1\right)}, \quad 0<u<1,
$$

which is a unimodal function of $u$ for $1<k<R_{i}$. The $100(1-\tau) \%$ height conditional density (HCD) prediction limits for $y$ are obtained as 


$$
L_{2}(x)=\left[-\frac{1}{\sqrt{\theta}} \Phi^{-1}\left(\frac{F^{*}}{2}\left(1-v_{1}\right)\right)\right]^{\frac{1}{\alpha}}, \quad U_{2}(x)=\left[-\frac{1}{\sqrt{\theta}} \Phi^{-1}\left(\frac{F^{*}}{2}\left(1-v_{2}\right)\right)\right]^{\frac{1}{\alpha}}
$$

where $v_{1}$ and $v_{2}$ are simultaneous solutions of the following equations

$$
\int_{v_{1}}^{v_{2}} g(u) d u=1-\tau
$$

and

$$
g\left(v_{1}\right)=g\left(v_{2}\right)
$$

We simplify equations (4.13) and (4.14) as

$$
B_{v_{2}}\left(k, R_{i}-k+1\right)-B_{v_{1}}\left(k, R_{i}-k+1\right)=1-\tau,
$$

and

$$
\left(\frac{1-v_{2}}{1-v_{1}}\right)^{R_{i}-k}=\left(\frac{v_{1}}{v_{2}}\right)^{k-1}
$$

where $B_{t}(p, q)=\frac{1}{B(p, q)} \int_{0}^{t} x^{p-1}(1-x)^{q-1} d x$ denotes the incomplete beta function.

\subsubsection{Bayesian Prediction Interval}

We first obtain a priori survival function and based on it, the desired predictive survival function can be obtained. Note that the a priori survival function is of the form

$$
S(y \mid x, \alpha, \theta)=\frac{\sum_{j=0}^{k-1}(-1)^{k-1-j} \frac{\left(1-F\left(x_{i}\right)\right)^{j-R_{i}(1-F(y))^{k-1-j}}}{R_{i}-j}}{\sum_{j=0}^{k-1} \frac{(-1)^{k-1-j}}{R_{i}-j}} .
$$

The posterior predictive survival function then turns out to be

$$
\hat{S}(y \mid x)=\int_{0}^{\infty} \int_{0}^{\infty} S(y \mid x, \alpha, \theta) \pi(\alpha, \theta \mid x) d \alpha d \theta .
$$

Finally, the two-sided $100(1-\tau) \%$ symmetric prediction interval $(L, U)$ of $y$ is obtained from the following two equations

$$
\hat{S}(L \mid x)=1-\frac{\tau}{2}, \quad \text { and } \hat{S}(U \mid x)=\frac{\tau}{2} .
$$




\section{Simulation Study and Data Analysis}

In this section, we compare the performance of the proposed methods using Monte Carlo simulations based on type II progressive hybrid censored data. The comparison of different point estimators of $\alpha$ and $\theta$ is made on the basis of their bias and mean square error (MSE) values. We compute these estimates for different censoring schemes by taking into account various $n, m$ and $T$ values. The bias and MSEs of each estimator are computed based on 5000 replications drawn from the $\operatorname{GHN}(0.5,1)$ distribution. The maximum likelihood estimates of parameters are computed using the EM algorithm.

We have performed all the computations on $R$ statistical software. The Bayes estimates are obtained with respect to the gamma prior distribution where hyperparameters are assigned as $a=2, b=4, p=3$ and $q=3$. We compute Bayes estimates of both the unknown parameters using TK and importance sampling procedures.

In Table 1, we have listed various censoring schemes for easy reference. Bias and MSEs of proposed estimators are presented in Tables 2-5 for two different choices of $T$ under the considered censoring schemes. For a given censoring scheme each cell of these tables contains four values. The first value denotes the estimate of $\alpha$ and the immediate lower value is the corresponding MSE. The last two values similarly represent the estimate of $\theta$ and the corresponding MSE. From these tables, we observe that the Bayes estimates of both the unknown parameters perform quite good compared to the respective MLEs. Both TK and importance sampling methods provide good estimation results. The TK estimates seem to have an advantage over the importance sampling estimates as it is computationally less intensive. Further, we get better estimation results with an increase in effective sample sizes. For fixed $n$ and $m$ values no specific trend is observed with an increase in $T$ values.

In Tables 6 and 7, we have presented asymptotic and HPD confidence intervals of both the unknown parameters $\alpha$ and $\theta$ for considered schemes. Again for a given censoring scheme each cell contains four values. The first two estimates respectively denote the average interval length (AL) and corresponding coverage probabilities (CP) associated with the parameter $\alpha$. Similarly, the last two estimates are for the parameter $\theta$. We mention that asymptotic intervals are obtained using the variance-covariance matrix of MLEs and HPD intervals are constructed with respect to the gamma prior distribution as discussed above. We compare these intervals in terms of their average length. From the tabulated estimates, we observe that HPD intervals of both the unknown parameters are narrower than the corresponding asymptotic intervals. In general theaverage interval length tend to decrease with an increase in effective sample 
size. Here also no specific trend is observed when $T$ is allowed to increase keeping $n$ and $m$ as fixed. The coverage probabilities of both asymptotic and HPD intervals are quite satisfactory and tend to remain close to the nominal $95 \%$ level.

In Table 8, we have computed all the prediction estimates of the first two observations censored at different stages $i$ before time $T$ when $T=0.64$ and $T=0.84$ under all the four schemes $S_{i}, i=1,2 \ldots 4$. We observe that in general BUP estimates are larger than CMP estimates followed by the Bayesian predictive estimates. The corresponding prediction results of the observations censored after time $\mathrm{T}$ are reported in Table 9 for different values of $R_{j}^{*}$ where $R_{j}^{*}=(c, d)$ implies that $R_{j}^{*}=c$ for the time $T=0.64$ and $R_{j}^{*}=d$ for the time $T=0.84$. From this table, we observe similar behavior that the Bayesian predictive estimates are in general smaller than the other two predictive estimates. Prediction intervals of the observations censored at different stages before the time $T$ are reported in Table 10. It is seen that the proposed prediction intervals behave quite similarly, however, the Bayes prediction interval has an advantage over the other two intervals as far as length is concerned. In general, prediction intervals tend to become wider for higher order censored observations. Finally, in Table 11, we have tabulated prediction intervals of observations censored after time $T$ and similar behavior is reported from this table as well.

Table 1: Removal patterns of units in various censoring schemes.

\begin{tabular}{lllll}
\hline \hline$(\mathrm{n}, \mathrm{m})$ & $S_{1}$ & $S_{2}$ & $S_{3}$ & $S_{4}$ \\
\hline \hline$(30,10)$ & $\left(20,0^{* 9}\right)$ & $\left(2^{* 10}\right)$ & $\left(2^{* 4}, 3^{* 3}, 1^{* 3}\right)$ & $\left(0^{* 9}, 20\right)$ \\
\hline$(30,15)$ & $\left(15,0^{* 14}\right)$ & $\left(1^{* 15}\right)$ & $\left(2^{* 3}, 3^{* 3}, 0^{* 9}\right)$ & $\left(0^{* 14}, 15\right)$ \\
\hline$(40,20)$ & $\left(20,0^{* 19}\right)$ & $\left(1^{* 20}\right)$ & $\left(2^{* 5}, 3^{* 3}, 1,0^{* 11}\right)$ & $\left(0^{* 19}, 20\right)$ \\
\hline$(40,30)$ & $\left(10,0^{* 29}\right)$ & $\left(2^{* 5}, 0^{* 25}\right)$ & $\left(2^{* 2}, 3^{* 2}, 0^{* 26}\right)$ & $\left(0^{* 29}, 10\right)$ \\
\hline
\end{tabular}




\begin{tabular}{|c|c|c|c|c|c|c|c|}
\hline $\bar{\infty}$ & $\bar{\omega}$ & 궁 & 겆 & $\underset{\infty}{\gtrless}$ & $\underset{\substack{\text { Ti } \\
2}}{\lessgtr}$ & & \\
\hline $\begin{array}{l}0 \\
\dot{0} \\
\dot{0} \\
\dot{0} \\
0 \\
0\end{array}$ & 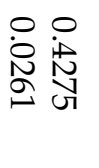 & 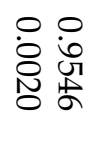 & 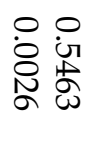 & $\stackrel{\circ}{\ominus} \underset{ٍ}{\ominus}$ & 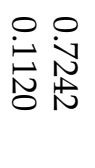 & $\omega$ & \\
\hline 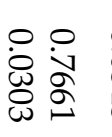 & 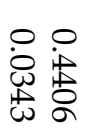 & 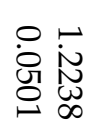 & $\begin{array}{ll}0 & 0 \\
\dot{8} & \text { iी } \\
\infty & 8 \\
\infty & 8 \\
0\end{array}$ & $\begin{array}{l}\stackrel{\circ}{\stackrel{N}{*}} \\
\stackrel{\circ}{\sigma}\end{array}$ & 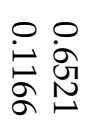 & $N$ & $\begin{array}{l}11 \\
0 \\
\dot{\phi} \\
\end{array}$ \\
\hline 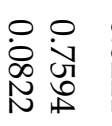 & 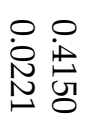 & 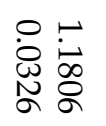 & 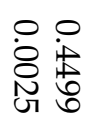 & 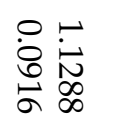 & 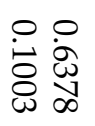 & $\infty$ & \\
\hline 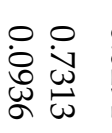 & 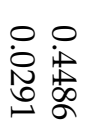 & 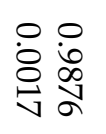 & 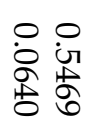 & 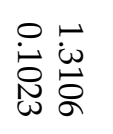 & 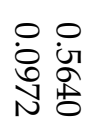 & $\infty$ & \\
\hline 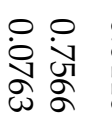 & 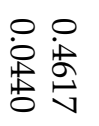 & 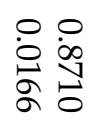 & 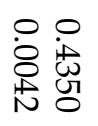 & 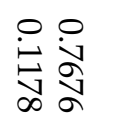 & $\begin{array}{l}\circ \stackrel{\circ}{\circ} \\
\stackrel{0}{\mathcal{N}} \\
\stackrel{N}{N}\end{array}$ & $\omega$ & $\rightarrow$ \\
\hline 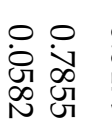 & 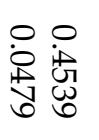 & 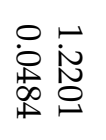 & 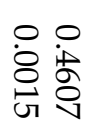 & 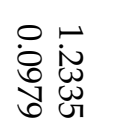 & 点点 & $N$ & \\
\hline 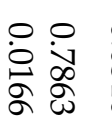 & 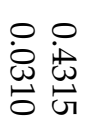 & 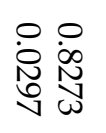 & $\begin{array}{l}\circ \\
\dot{8} \\
\stackrel{i}{\infty} \\
\stackrel{\infty}{\infty} \\
\stackrel{\infty}{\infty}\end{array}$ & 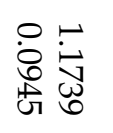 & 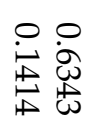 & $\omega$ & \\
\hline 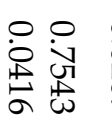 & 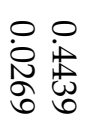 & $\begin{array}{l}\circ \\
\dot{8} \\
\dot{0} \\
\dot{1} \\
\infty\end{array}$ & 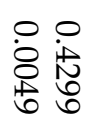 & $\begin{array}{l}\stackrel{\circ}{\vec{\omega}} \\
\text { 芯芯 }\end{array}$ & 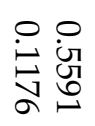 & $\omega$ & \\
\hline
\end{tabular}




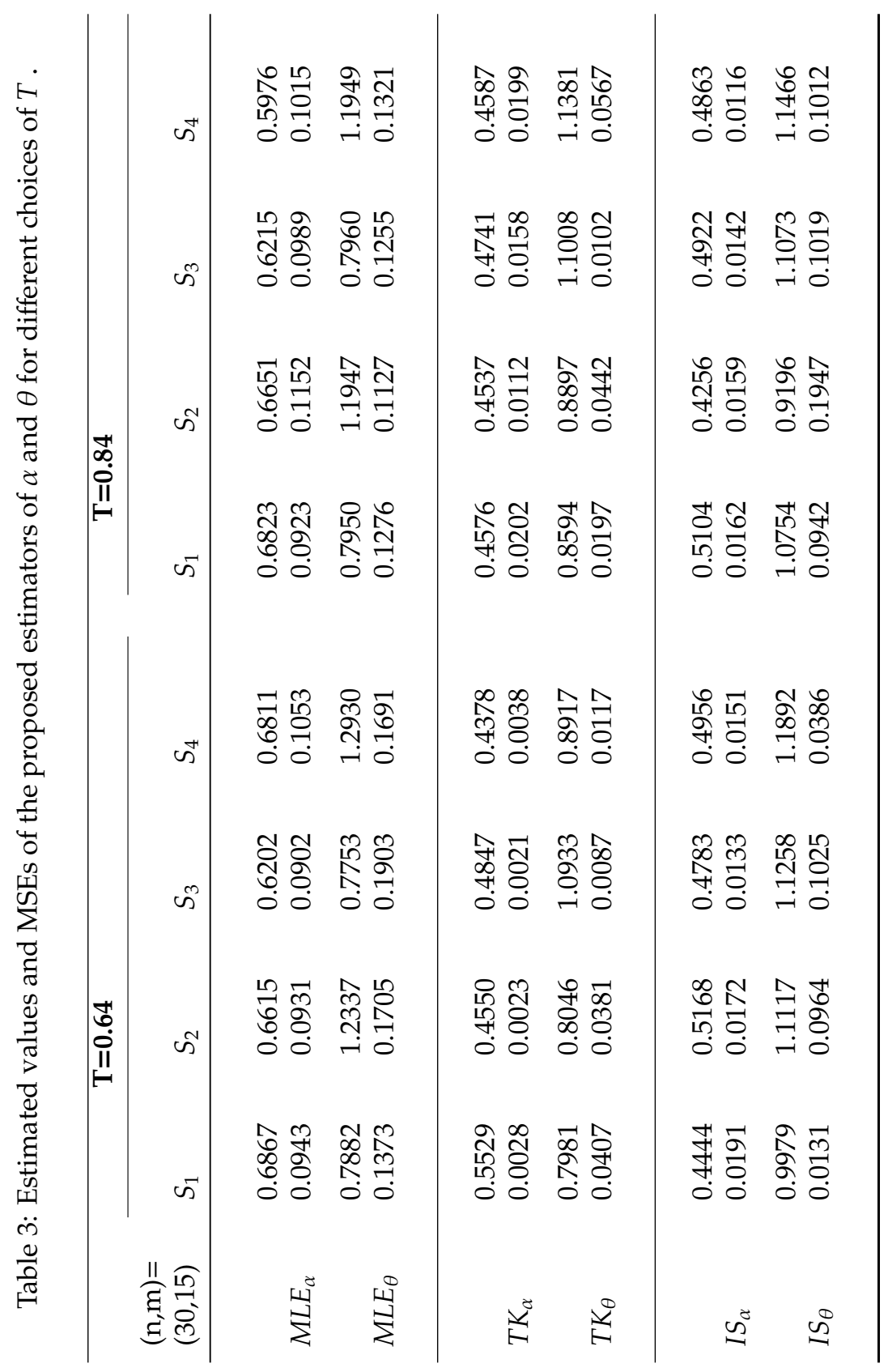




\begin{tabular}{|c|c|c|c|c|c|c|c|}
\hline is & $\bar{\varpi}$ & $\underset{\Theta}{-ત}$ & $\overrightarrow{ }$ & $\underset{0}{\gtrless}$ & 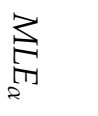 & $\begin{array}{l}\text { 在 } \\
\text { O } \\
0\end{array}$ & \\
\hline 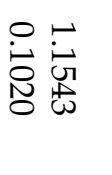 & 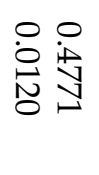 & 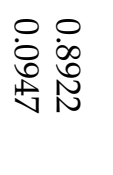 & 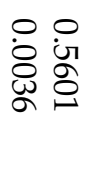 & 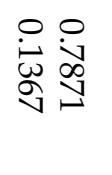 & 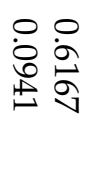 & $\omega$ & \\
\hline 递曷 & 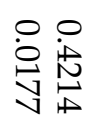 & 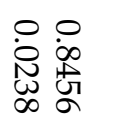 & 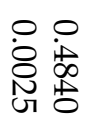 & $\stackrel{\circ}{\stackrel{N}{N}}$ & $\begin{array}{l}\circ \stackrel{0}{\circ} \\
\dot{\sigma} \\
\sigma \\
\sigma\end{array}$ & N & $\begin{array}{l}11 \\
\text { II } \\
\dot{1}\end{array}$ \\
\hline 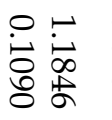 & 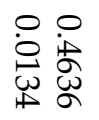 & 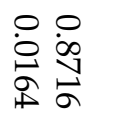 & 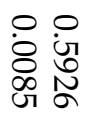 & 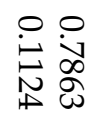 & 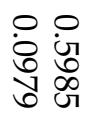 & $\omega$ & \\
\hline$\stackrel{\circ}{\stackrel{\vec{\sigma}}{\overrightarrow{0}}}$ & 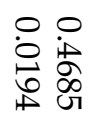 & 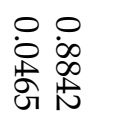 & 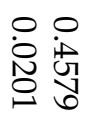 & $\begin{array}{l}\stackrel{\circ}{\vec{N}} \\
\stackrel{\sim}{N} \\
\infty\end{array}$ & 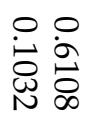 & $\infty$ & \\
\hline$\stackrel{\circ}{\stackrel{\bullet}{\vec{\omega}}}$ & 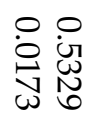 & 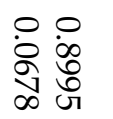 & 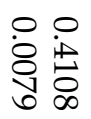 & 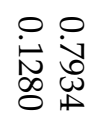 & 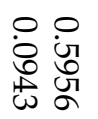 & $\omega$ & -7 \\
\hline 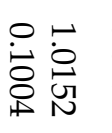 & 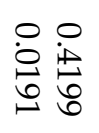 & 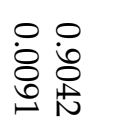 & 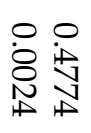 & 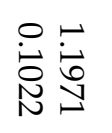 & 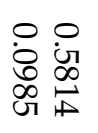 & $N$ & \\
\hline 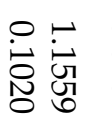 & 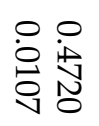 & 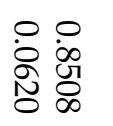 & $\begin{array}{l}0 \\
\dot{0} \\
8 \\
0 \\
0\end{array}$ & 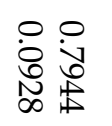 & $\begin{array}{l}0 \\
\stackrel{0}{0} \\
\dot{0} \\
0 \\
0 \\
0\end{array}$ & $\omega$ & \\
\hline $\begin{array}{l}\circ \\
\dot{0} \\
\stackrel{0}{0}\end{array}$ & 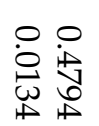 & 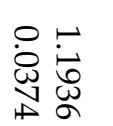 & 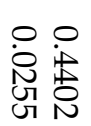 & 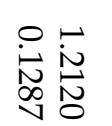 & $\begin{array}{l}\circ 0 \\
\dot{0}: \\
\dot{0} \\
\varnothing \\
\infty\end{array}$ & $\stackrel{\omega}{\infty}$ & \\
\hline
\end{tabular}




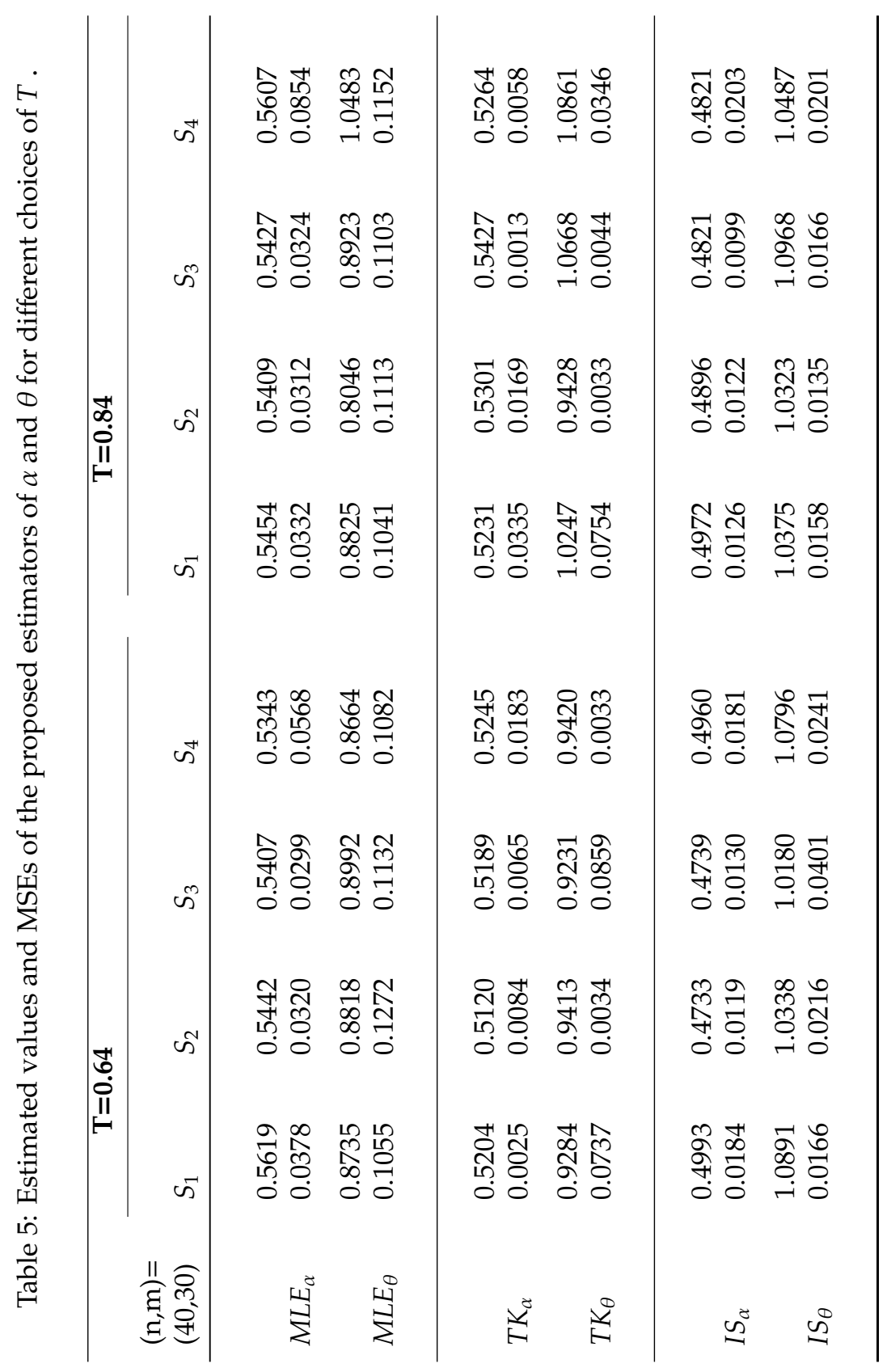




\begin{tabular}{|c|c|c|c|c|c|c|c|c|c|}
\hline 0 & 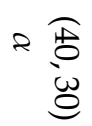 & 0 & $\begin{array}{r}2 \text { 居 } \\
\text { N }\end{array}$ & 0 & 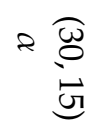 & 0 & 2 & $\begin{array}{l}0 \\
0 \\
0 \\
0 \\
0\end{array}$ & \\
\hline 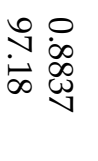 & 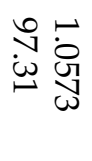 & 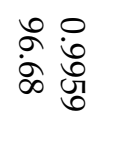 & ஜํ. & 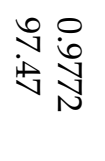 & 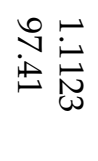 & 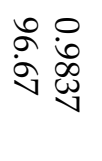 & 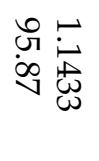 & $\infty$ & 萑 \\
\hline 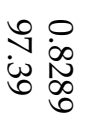 & 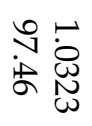 & $\begin{array}{l}\text { 요 } \\
\text { o̊ } \\
\text { vo } \\
\sigma\end{array}$ & 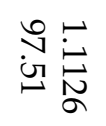 & 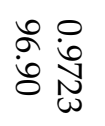 & 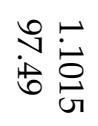 & 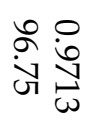 & 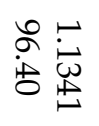 & 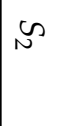 & 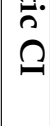 \\
\hline 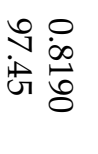 & 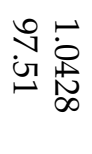 & 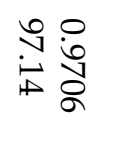 & $\begin{array}{l}\stackrel{v}{*} \dot{8} \\
\text { ஸु }\end{array}$ & 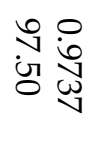 & 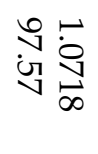 & 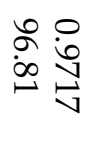 & 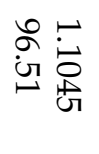 & $\infty$ & \\
\hline 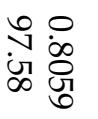 & 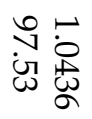 & 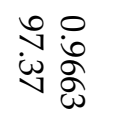 & 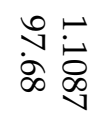 & 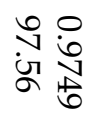 & $\begin{array}{l}\stackrel{0}{\vec{N}} \\
\dot{\omega} \\
\stackrel{N}{a}\end{array}$ & 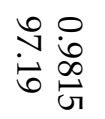 & 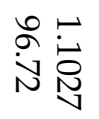 & $\infty$ & \\
\hline 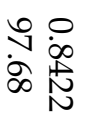 & 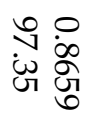 & 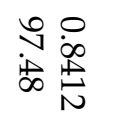 & 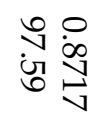 & 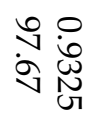 & 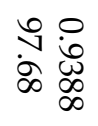 & 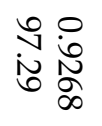 & 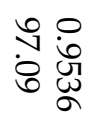 & $\infty$ & \\
\hline 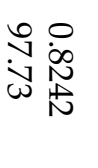 & 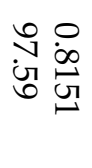 & 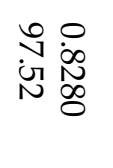 & 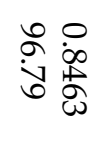 & 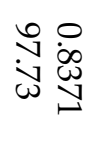 & 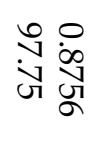 & 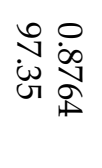 & $\stackrel{\circ}{\stackrel{\circ}{ }} \stackrel{\circ}{\circ}$ & N & $\begin{array}{l}\bar{\tau} \\
\boldsymbol{\theta} \\
\tilde{\sigma}\end{array}$ \\
\hline $\begin{array}{l}0 \\
\stackrel{0}{\circ} \\
\infty \\
\infty \\
+ \\
\stackrel{0}{0}\end{array}$ & 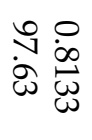 & 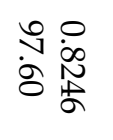 & 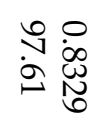 & \begin{tabular}{ll}
0 & 0 \\
1 & $\infty$ \\
$\infty$ & $\omega$ \\
$\triangleright$ & \multicolumn{1}{|c}{}
\end{tabular} & 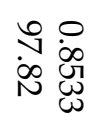 & 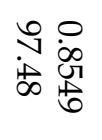 & 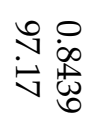 & $\infty$ & \\
\hline 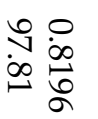 & 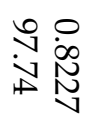 & $\begin{array}{l}0 \\
\stackrel{\circ}{\infty} \\
\dot{\infty} \stackrel{\infty}{\omega} \\
\sigma\end{array}$ & 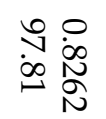 & $\begin{array}{l}0 \\
\infty \\
\infty \\
\dot{0} \\
\dot{0}\end{array}$ & 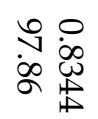 & 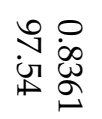 & 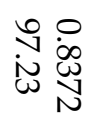 & $\underset{\oplus}{\infty}$ & \\
\hline
\end{tabular}




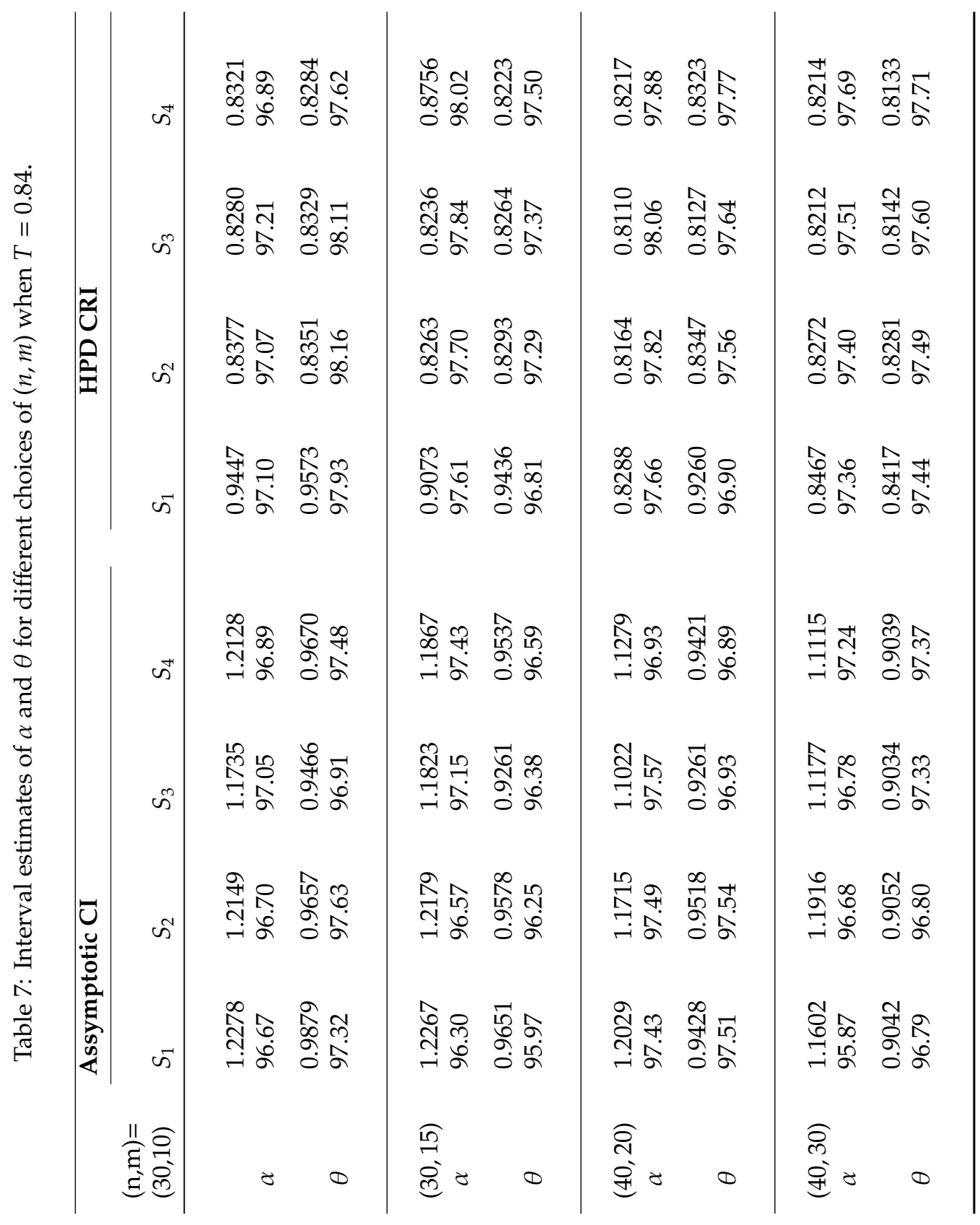


Table 8: Prediction estimates for observations censored before time $T$.

\begin{tabular}{|c|c|c|c|c|c|c|c|c|c|}
\hline \multirow[b]{2}{*}{$(n, m)$} & \multirow[b]{2}{*}{ Scheme } & \multicolumn{5}{|c|}{$T=0.64$} & \multicolumn{3}{|c|}{$T=0.84$} \\
\hline & & $\mathrm{i}$ & $\mathrm{k}$ & MLP & BUP & $\mathrm{BP}$ & MLP & BUP & $\mathrm{BP}$ \\
\hline \multirow[t]{8}{*}{$(30,10)$} & \multirow[t]{2}{*}{$S_{1}$} & \multirow{2}{*}{1} & 1 & 0.4152 & 0.4157 & 0.3641 & 0.4122 & 0.4127 & 0.3580 \\
\hline & & & 2 & 0.4509 & 0.4518 & 0.4371 & 0.4177 & 0.4212 & 0.3979 \\
\hline & \multirow[t]{2}{*}{$S_{2}$} & \multirow[t]{2}{*}{2} & 1 & 0.4067 & 0.4117 & 0.4020 & 0.4127 & 0.4208 & 0.4015 \\
\hline & & & 2 & 0.4255 & 0.4309 & 0.4217 & 0.4206 & 0.4233 & 0.4177 \\
\hline & \multirow[t]{2}{*}{$S_{3}$} & \multirow[t]{2}{*}{5} & 1 & 0.4217 & 0.4236 & 0.4162 & 0.4175 & 0.4183 & 0.4144 \\
\hline & & & 2 & 0.4288 & 0.4296 & 0.4211 & 0.4231 & 0.4254 & 0.4206 \\
\hline & \multirow[t]{2}{*}{$S_{4}$} & \multirow[t]{2}{*}{10} & 1 & 0.4338 & 0.4362 & 0.4307 & 0.4293 & 0.4406 & 0.4288 \\
\hline & & & 2 & 0.4418 & 0.4434 & 0.4669 & 0.4323 & 0.4347 & 0.4311 \\
\hline \multirow[t]{7}{*}{$(30,15)$} & \multirow[t]{2}{*}{$S_{1}$} & \multirow[t]{2}{*}{1} & 1 & 0.4220 & 0.4239 & 0.4144 & 0.4187 & 0.4204 & 0.4125 \\
\hline & & & 2 & 0.4263 & 0.4291 & 0.4220 & 0.4212 & 0.4229 & 0.4193 \\
\hline & $S_{2}$ & 1 & 1 & 0.4118 & 0.4477 & 0.4380 & 0.4407 & 0.4420 & 0.4370 \\
\hline & \multirow[t]{2}{*}{$S_{3}$} & \multirow[t]{2}{*}{4} & 1 & 0.4437 & 0.4456 & 0.4412 & 0.4410 & 0.4419 & 0.4391 \\
\hline & & & 2 & 0.4521 & 0.4534 & 0.4506 & 0.4432 & 0.4449 & 0.4408 \\
\hline & \multirow[t]{2}{*}{$S_{4}$} & \multirow[t]{2}{*}{15} & 1 & 0.4533 & 0.4549 & 0.4512 & 0.4488 & 0.4511 & 0.4470 \\
\hline & & & 2 & 0.4621 & 0.4641 & 0.4607 & 0.4535 & 0.4577 & 0.4518 \\
\hline \multirow[t]{7}{*}{$(40,20)$} & \multirow[t]{2}{*}{$S_{1}$} & \multirow[t]{2}{*}{1} & 1 & 0.5077 & 0.5084 & 0.5011 & 0.5019 & 0.5048 & 0.5006 \\
\hline & & & 2 & 0.5169 & 0.5204 & 0.5143 & 0.5131 & 0.5163 & 0.5117 \\
\hline & $S_{2}$ & 1 & 1 & 0.5155 & 0.5172 & 0.5133 & 0.5125 & 0.5137 & 0.5119 \\
\hline & \multirow[t]{2}{*}{$S_{3}$} & \multirow[t]{2}{*}{6} & 1 & 0.5227 & 0.5241 & 0.5207 & 0.5174 & 0.5186 & 0.5163 \\
\hline & & & 2 & 0.5376 & 0.5387 & 0.5341 & 0.5255 & 0.5283 & 0.5239 \\
\hline & \multirow[t]{2}{*}{$S_{4}$} & \multirow[t]{2}{*}{20} & 1 & 0.5561 & 0.5583 & 0.5529 & 0.5453 & 0.5476 & 0.5420 \\
\hline & & & 2 & 0.5601 & 0.5628 & 0.5591 & 0.5529 & 0.5563 & 0.5511 \\
\hline \multirow[t]{8}{*}{$(40,30)$} & $S_{1}$ & 1 & 1 & 0.5695 & 0.5711 & 0.5680 & 0.5477 & 0.5485 & 0.5468 \\
\hline & & & 2 & 0.5727 & 0.5739 & 0.5710 & 0.5597 & 0.5608 & 0.5581 \\
\hline & $S_{2}$ & 2 & 1 & 0.5713 & 0.5728 & 0.5697 & 0.5743 & 0.5786 & 0.5711 \\
\hline & & & 2 & 0.5729 & 0.5747 & 0.5710 & 0.5759 & 0.5777 & 0.5718 \\
\hline & $S_{3}$ & 3 & 1 & 0.5788 & 0.5804 & 0.5740 & 0.5769 & 0.5798 & 0.5725 \\
\hline & & & 2 & 0.5796 & 0.5817 & 0.5761 & 0.5790 & 0.5830 & 0.5754 \\
\hline & $S_{4}$ & 30 & 1 & 0.5869 & 0.5890 & 0.5840 & 0.5856 & 0.5887 & 0.5829 \\
\hline & & & 2 & 0.5876 & 0.5911 & 0.5866 & 0.5863 & 0.5873 & 0.5841 \\
\hline
\end{tabular}


Table 9: Prediction estimates for observations censored after time $T$.

\begin{tabular}{|c|c|c|c|c|c|c|c|c|c|}
\hline \multirow[b]{2}{*}{$(n, m)$} & \multirow[b]{2}{*}{ Scheme } & \multicolumn{5}{|c|}{$T=0.64$} & \multicolumn{3}{|c|}{$T=0.84$} \\
\hline & & $R_{j} *$ & $\mathrm{k}$ & MLP & BUP & $\mathrm{BP}$ & MLP & BUP & $\mathrm{BP}$ \\
\hline \multirow[t]{8}{*}{$(30,10)$} & \multirow[t]{2}{*}{$S_{1}$} & \multirow{2}{*}{$(5,3)$} & 1 & 1.6734 & 1.7822 & 1.4211 & 1.8130 & 1.8670 & 1.3951 \\
\hline & & & 2 & 1.7346 & 1.8742 & 1.4719 & 1.6970 & 1.7349 & 1.4466 \\
\hline & \multirow[t]{2}{*}{$S_{2}$} & \multirow[t]{2}{*}{$(6,4)$} & 1 & 1.7225 & 1.8395 & 1.4287 & 1.7460 & 1.8612 & 1.4291 \\
\hline & & & 2 & 1.7628 & 1.8742 & 1.4739 & 1.7840 & 1.9373 & 1.5166 \\
\hline & \multirow[t]{2}{*}{$S_{3}$} & \multirow[t]{2}{*}{$(6,2)$} & 1 & 1.7232 & 1.8035 & 1.4276 & 1.7421 & 1.8408 & 1.4701 \\
\hline & & & 2 & 1.7611 & 1.8742 & 1.4739 & 1.8270 & 1.9753 & 1.5618 \\
\hline & \multirow[t]{2}{*}{$S_{4}$} & \multirow[t]{2}{*}{$(6,3)$} & 1 & 1.7333 & 1.7953 & 1.4270 & 1.7493 & 1.8408 & 1.4439 \\
\hline & & & 2 & 1.7610 & 1.8742 & 1.4731 & 1.7767 & 1.8783 & 1.4868 \\
\hline \multirow[t]{7}{*}{$(30,15)$} & \multirow[t]{2}{*}{$S_{1}$} & \multirow[t]{2}{*}{$(7,4)$} & 1 & 1.7321 & 1.7785 & 1.5711 & 1.7423 & 1.7914 & 1.5901 \\
\hline & & & 2 & 1.7367 & 1.8142 & 1.5769 & 1.7609 & 1.8603 & 1.6118 \\
\hline & $S_{2}$ & $(9,4)$ & 1 & 1.7392 & 1.8335 & 1.5371 & 1.7603 & 1.8411 & 1.5396 \\
\hline & \multirow[t]{2}{*}{$S_{3}$} & \multirow[t]{2}{*}{$(9,3)$} & 1 & 1.7433 & 1.8143 & 1.5427 & 1.7573 & 1.8406 & 1.5905 \\
\hline & & & 2 & 1.7613 & 1.8426 & 1.5629 & 1.8170 & 1.8733 & 1.5768 \\
\hline & \multirow[t]{2}{*}{$S_{4}$} & \multirow[t]{2}{*}{$(10,6)$} & 1 & 1.7462 & 1.8245 & 1.5417 & 1.7640 & 1.8409 & 1.5690 \\
\hline & & & 2 & 1.7569 & 1.8712 & 1.5709 & 1.7705 & 1.8693 & 1.5928 \\
\hline \multirow[t]{7}{*}{$(40,20)$} & \multirow[t]{2}{*}{$S_{1}$} & \multirow[t]{2}{*}{$(13,10)$} & 1 & 1.8431 & 1.8892 & 1.6037 & 1.940 & 2.1148 & 1.6351 \\
\hline & & & 2 & 1.8729 & 1.9174 & 1.6179 & 1.9216 & 1.9773 & 1.6228 \\
\hline & $S_{2}$ & $(12,9)$ & 1 & 1.8472 & 1.9235 & 1.6217 & 1.93463 & 2.0867 & 1.6352 \\
\hline & \multirow[t]{2}{*}{$S_{3}$} & \multirow[t]{2}{*}{$(11,8)$} & 1 & 1.8447 & 1.9351 & 1.6421 & 1.9723 & 2.1522 & 1.6538 \\
\hline & & & 2 & 1.8736 & 2.1142 & 1.6579 & 1.9770 & 2.1639 & 1.6608 \\
\hline & \multirow[t]{2}{*}{$S_{4}$} & \multirow[t]{2}{*}{$(13,9)$} & 1 & 1.8392 & 1.9523 & 1.6433 & 1.9403 & 2.1048 & 1.6641 \\
\hline & & & 2 & 1.8731 & 2.1402 & 1.6721 & 1.9360 & 2.1669 & 1.6825 \\
\hline \multirow[t]{8}{*}{$(40,30)$} & $S_{1}$ & $(12,8)$ & 1 & 1.9142 & 2.1325 & 1.6732 & 1.9703 & 2.1751 & 1.6910 \\
\hline & & & 2 & 2.0365 & 2.1832 & 1.6908 & 2.1720 & 2.2173 & 1.6892 \\
\hline & $S_{2}$ & $(9,6)$ & 1 & 1.8255 & 1.9430 & 1.6734 & 1.9333 & 2.0834 & 1.6911 \\
\hline & & & 2 & 1.8738 & 2.1412 & 1.6792 & 2.0917 & 2.1731 & 1.6843 \\
\hline & $S_{3}$ & $(12,8)$ & 1 & 1.9244 & 2.1455 & 1.6907 & 2.1039 & 2.1865 & 1.7123 \\
\hline & & & 2 & 2.0346 & 2.1760 & 1.7209 & 2.1580 & 2.2363 & 1.7301 \\
\hline & $S_{4}$ & $(10,6)$ & 1 & 2.0326 & 2.1544 & 1.7276 & 2.1411 & 2.1867 & 1.7391 \\
\hline & & & 2 & 2.1906 & 2.2462 & 1.7416 & 2.2260 & 2.2790 & 1.7603 \\
\hline
\end{tabular}




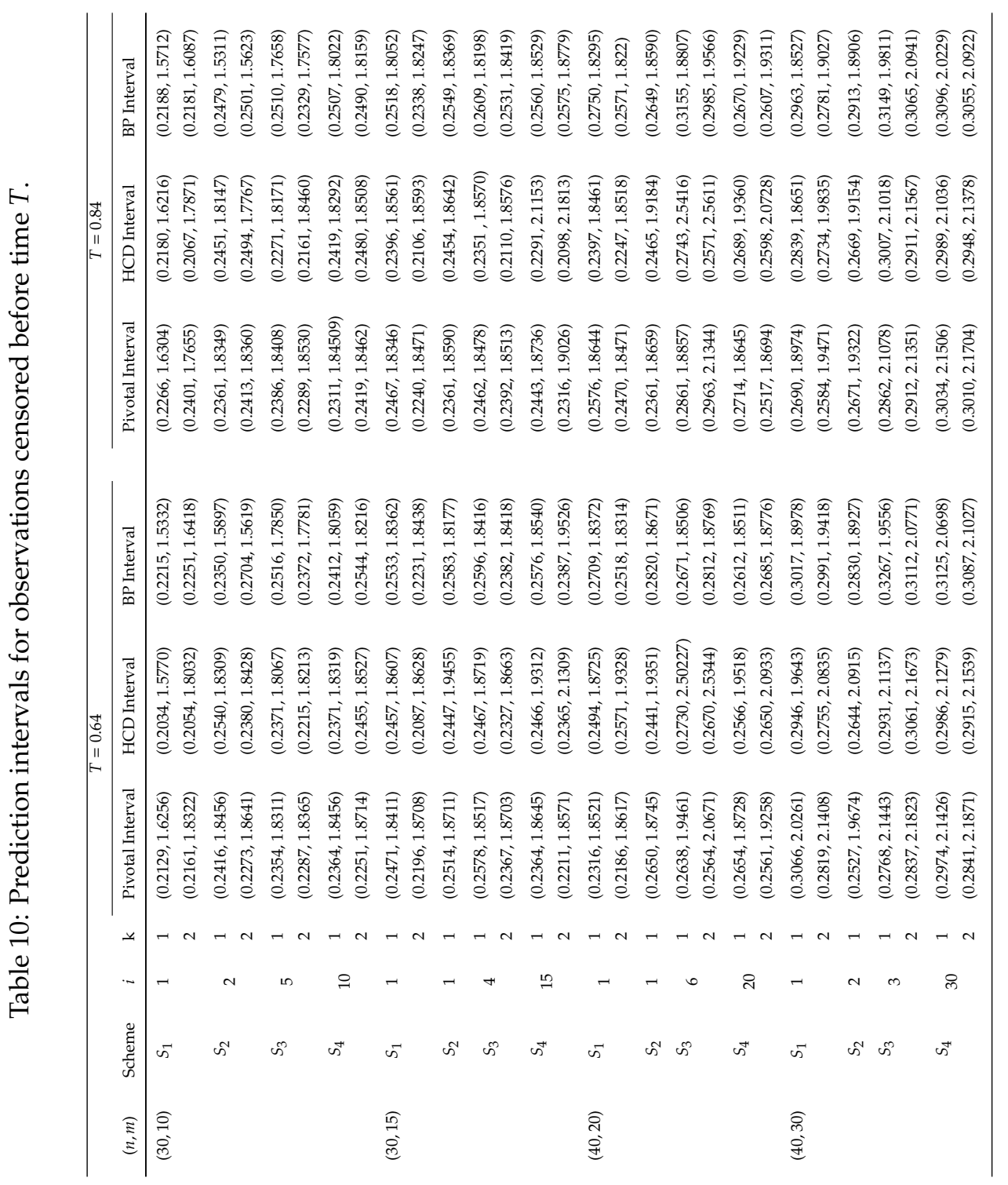




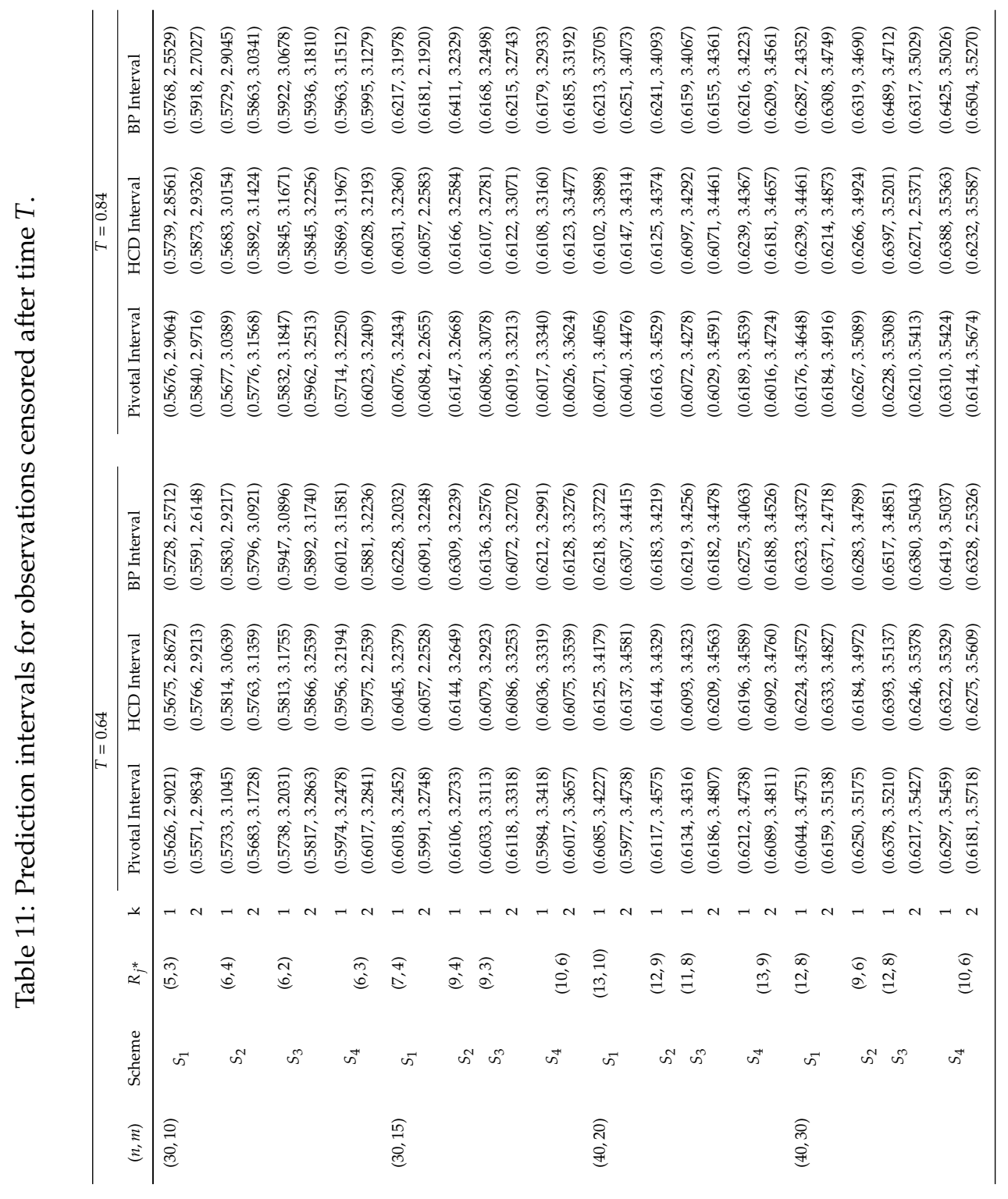




\subsection{Real Data Analysis}

Here we analyze a real data set in support of the proposed methods. This data set is discussed in Andrews and Herzberg (1985) (see also, Barlow et al. (1984)) and it represents the stress-rupture life of kevlar 49/epoxy strands which were subjected to constant sustained pressure at the $90 \%$ stress level. The failure times in hours are recorded as

$0.01,0.01,0.02,0.02,0.02,0.03,0.03,0.04,0.05,0.06,0.07,0.07,0.08$, $0.09,0.09,0.10,0.10,0.11,0.11,0.12,0.13,0.18,0.19,0.20,0.23,0.24$, $0.24,0.29,0.34,0.35,0.36,0.38,0.40,0.42,0.43,0.52,0.54,0.56,0.60$, $0.60,0.63,0.65,0.67,0.68,0.72,0.72,0.72,0.73,0.79,0.79,0.80,0.80$, $0.83,0.85,0.90,0.92,0.95,0.99,1.00,1.01,1.02,1.03,1.05,1.10,1.10$, $1.11,1.15,1.18,1.20,1.29,1.31,1.33,1.34,1.40,1.43,1.45,1.50,1.51$, $1.52,1.53,1.54,1.54,1.55,1.58,1.60,1.63,1.64,1.80,1.80,1.81,2.02$, $2.05,2.14,2.17,2.33,3.03,3.03,3.34,4.20,4.69,7.89$.

We first verify whether a $G H N(\alpha ; \theta)$ distribution is suitable for making inference from this data. We fit the data using four different distributions, namely generalized half-normal, generalized exponential, Weibull and Burr XII. We judge the goodness of fit of competing models using different criteria.

In Table 12, we present MLEs of unknown parameters of all distributions along with estimated values of Akaike's information criterion (AIC) and the associated second order information criterion (AICc), Bayesian information criterion (BIC) and the negative log-likelihood criterion. From this table, we observe that the GHN distribution fits the data set reasonably good compared to the other models. So, we make inference from the given data set using this distribution. Two different censoring schemes $S_{1}=\left(0^{* 13}, 3^{* 7}, 4^{* 5}, 2^{* 10}, 0^{* 5}\right)$ and $S_{2}=\left(0^{* 5}, 3^{* 17}, 1^{* 10}, 0^{* 8}\right)$ are taken into consideration using $(n, m, T)$ values such that $S_{1}:(n, m)=(101,40) ; S_{2}:(n, m)=(101,40)$ and $T$ is either 0.36 or 0.52 . The generated sets under these schemes are respectively given by

$0.01,0.01,0.02,0.02,0.02,0.03,0.03,0.04,0.05,0.06,0.07,0.07,0.08$, $0.09,0.10,0.11,0.13,0.20,0.23,0.35,0.36,0.38,0.40,0.42,0.63,0.72$, $0.72,0.79,0.80,1.01,1.02,1.20,1.29,1.31,1.34,2.02,2.14,3.03,3.34,4.69$.

and

$0.01,0.01,0.02,0.02,0.02,0.03,0.03,0.05,0.07,0.09,0.11,0.13,0.18$, $0.24,0.29,0.36,0.40,0.42,0.60,0.63,0.67,0.68,0.72,0.72,0.73,0.80$, $0.85,1.01,1.20,1.29,1.31,1.43,1.54,1.54,1.60,1.81,2.02,2.17,3.03,7.89$. 
In Table 13, we report maximum likelihood and the Bayes estimates of the parameters $\alpha$ and $\theta$ for both of the censoring schemes. For each scheme, the first value denotes estimate of $\alpha$ and the immediate lower value is the estimate of the parameter $\theta$. The Bayes estimates are computed with respect to a noninformative prior distribution where all hyperparameters are assigned the zero value. It is seen that the estimated values of the respective parameters remain relatively close to each other.

The 95\% asymptotic and noninformative HPD intervals are reported in Table 14 for both of the censoring schemes. In general, asymptotic intervals are wider than respective noninformative HPD intervals. In Table 15, we have computed the prediction estimates of the first three observations censored at different stages $i$ before time $T$ which may be either 0.36 or 0.52 under both of the schemes $S_{1}$ and $S_{2}$. We observe that the predicted estimates from different methods remain satisfactorily close to each other. The corresponding prediction results of the observations censored after the time $T$ are reported in Table 16.

Prediction intervals of the first three observations censored at different stages before time $T$ are reported in Table 17. It is seen that the prediction intervals tend to become wider for higher order censored observations. Finally, in Table 18 we have tabulated the prediction intervals of observations censored after time $T$ and we report similar behavior from this table as well.

Table 12: Goodness of fit tests for different distributions.

\begin{tabular}{lllllll}
\hline & $\hat{\alpha}$ & $\hat{\theta}$ & $\begin{array}{l}\text { Negative } \\
\text { log- } \\
\text { likelihood }\end{array}$ & AIC & AICc & BIC \\
\hline GHN & 1.4845 & 1.1629 & 1.3243 & 6.6487 & 6.7712 & 11.8789 \\
GE & 6.6606 & 5.9998 & 12.4669 & 28.9337 & 29.0562 & 34.1639 \\
Weibull & 7.7119 & 5.9996 & 19.1898 & 42.3796 & 42.5020 & 47.6098 \\
Burr XII & 5.5737 & 5.9991 & 17.5531 & 39.1063 & 39.2288 & 44.3366 \\
\hline
\end{tabular}


Table 13: Estimates of $\alpha$ and $\theta$ for different choices of $T$.

\begin{tabular}{llll}
\hline & Scheme & $T=0.36$ & $T=0.52$ \\
\hline$E M$ & $S_{1}$ & 0.9527 & 0.8856 \\
& & 1.0122 & 1.1433 \\
& $S_{2}$ & 0.8195 & 0.8082 \\
& & 1.1329 & 1.2566 \\
\hline$T K$ & $S_{1}$ & 1.0812 & 0.7843 \\
& & 1.2164 & 1.1407 \\
& $S_{2}$ & 0.8069 & 0.8152 \\
& & 1.2218 & 1.1855 \\
\hline IS & $S_{1}$ & 0.8640 & 0.8174 \\
& & 1.1011 & 1.0779 \\
& & 0.8351 & 0.8847 \\
& $S_{2}$ & 1.0551 & 1.0478 \\
\hline
\end{tabular}

Table 14: Interval estimates of $\alpha$ and $\theta$ for different choices of $T$.

\begin{tabular}{lllllll}
\hline \multicolumn{3}{c}{$T=0.36$} & & & $T=0.52$ & \\
\cline { 1 - 2 } \cline { 5 - 6 } Scheme & Asymptotic CI & HPD CRI & & Asymptotic CI & HPD CRI \\
\hline$S_{1}$ & $(0.5788,1.2169)$ & $(0.8945,1.4772)$ & & $(0.6033,1.2530)$ & $(0.9254,1.4083)$ \\
& $(0.9248,1.8922)$ & $(0.7839,1.2043)$ & & $(0.9079,1.7841)$ & $(0.8263,1.1976)$ \\
\hline$S_{2}$ & $(0.4975,1.1361)$ & $(0.8459,1.3621)$ & & $(0.4761,1.2109)$ & $(0.7896,1.2985)$ \\
& $(0.8837,1.7393)$ & $(0.6896,1.1360)$ & & $(0.9117,1.6793)$ & $(0.7564,1.1468)$ \\
\hline
\end{tabular}


Table 15: Prediction estimates for observations censored before time $T$.

\begin{tabular}{|c|c|c|c|c|c|c|c|c|}
\hline \multirow[b]{2}{*}{ Scheme } & \multirow[b]{2}{*}{ i } & \multirow[b]{2}{*}{$\mathrm{k}$} & \multicolumn{3}{|c|}{$T=0.36$} & \multicolumn{3}{|c|}{$T=0.52$} \\
\hline & & & MLP & BUP & $\mathrm{BP}$ & MLP & BUP & $\mathrm{BP}$ \\
\hline \multirow{2}{*}{$S_{1}$} & \multirow[b]{2}{*}{14} & 1 & 0.1432 & 0.1435 & 0.1427 & 0.1403 & 0.1408 & 0.1391 \\
\hline & & 2 & 0.1736 & 0.1742 & 0.1739 & 0.1670 & 0.1673 & 0.1668 \\
\hline \multirow{10}{*}{$S_{2}$} & \multirow{4}{*}{21} & 3 & 0.1766 & 0.1769 & 0.1761 & 0.1738 & 0.1741 & 0.1737 \\
\hline & & 1 & 0.4150 & 0.4153 & 0.4147 & 0.4136 & 0.4237 & 0.4133 \\
\hline & & 2 & 0.4313 & 0.4317 & 0.4311 & 0.4307 & 0.4310 & 0.4305 \\
\hline & & 3 & 0.4390 & 0.4393 & 0.4391 & 0.4359 & 0.4361 & 0.4357 \\
\hline & \multirow{3}{*}{6} & 1 & 0.1232 & 0.1235 & 0.1229 & 0.1189 & 0.1191 & 0.1187 \\
\hline & & 2 & 0.1253 & 0.1255 & 0.1251 & 0.1250 & 0.1252 & 0.1248 \\
\hline & & 3 & 0.1306 & 0.1308 & 0.1305 & 0.1307 & 0.1309 & 0.1303 \\
\hline & \multirow{3}{*}{8} & 1 & 0.1220 & 0.1222 & 0.1217 & 0.1219 & 0.1221 & 0.1218 \\
\hline & & 2 & 0.1227 & 0.1230 & 0.1225 & 0.1226 & 0.1228 & 0.1223 \\
\hline & & 3 & 0.1234 & 0.1236 & 0.1233 & 0.1232 & 0.1235 & 0.1230 \\
\hline
\end{tabular}


Table 16: Prediction estimates for observations censored after time $T$.

\begin{tabular}{|c|c|c|c|c|c|c|}
\hline Scheme & $T$ & $R_{j}^{*}$ & $\mathrm{k}$ & MLP & BUP & $\mathrm{BP}$ \\
\hline \multirow{2}{*}{$S_{1}$} & \multirow{3}{*}{0.36} & \multirow{3}{*}{41} & 1 & 1.9088 & 1.9674 & 1.9745 \\
\hline & & & 2 & 1.9840 & 2.0731 & 2.1148 \\
\hline \multirow{4}{*}{$S_{1}$} & & & 3 & 2.2681 & 2.2945 & 2.4209 \\
\hline & \multirow{3}{*}{0.52} & \multirow{3}{*}{36} & 1 & 1.8771 & 1.9264 & 1.9941 \\
\hline & & & 2 & 2.1262 & 2.1645 & 2.2920 \\
\hline & & & 3 & 2.3211 & 2.2971 & 2.3904 \\
\hline \multirow{2}{*}{$S_{2}$} & \multirow{3}{*}{0.36} & \multirow{3}{*}{46} & 1 & 2.5067 & 2.6482 & 2.9841 \\
\hline & & & 2 & 2.6244 & 2.6951 & 3.0722 \\
\hline \multirow{4}{*}{$S_{2}$} & & & 3 & 2.8513 & 2.9741 & 3.0519 \\
\hline & \multirow{3}{*}{0.52} & \multirow{3}{*}{43} & 1 & 2.7514 & 2.8364 & 2.9807 \\
\hline & & & 2 & 3.1240 & 3.1802 & 3.2231 \\
\hline & & & 3 & 3.1523 & 3.2971 & 3.4229 \\
\hline
\end{tabular}




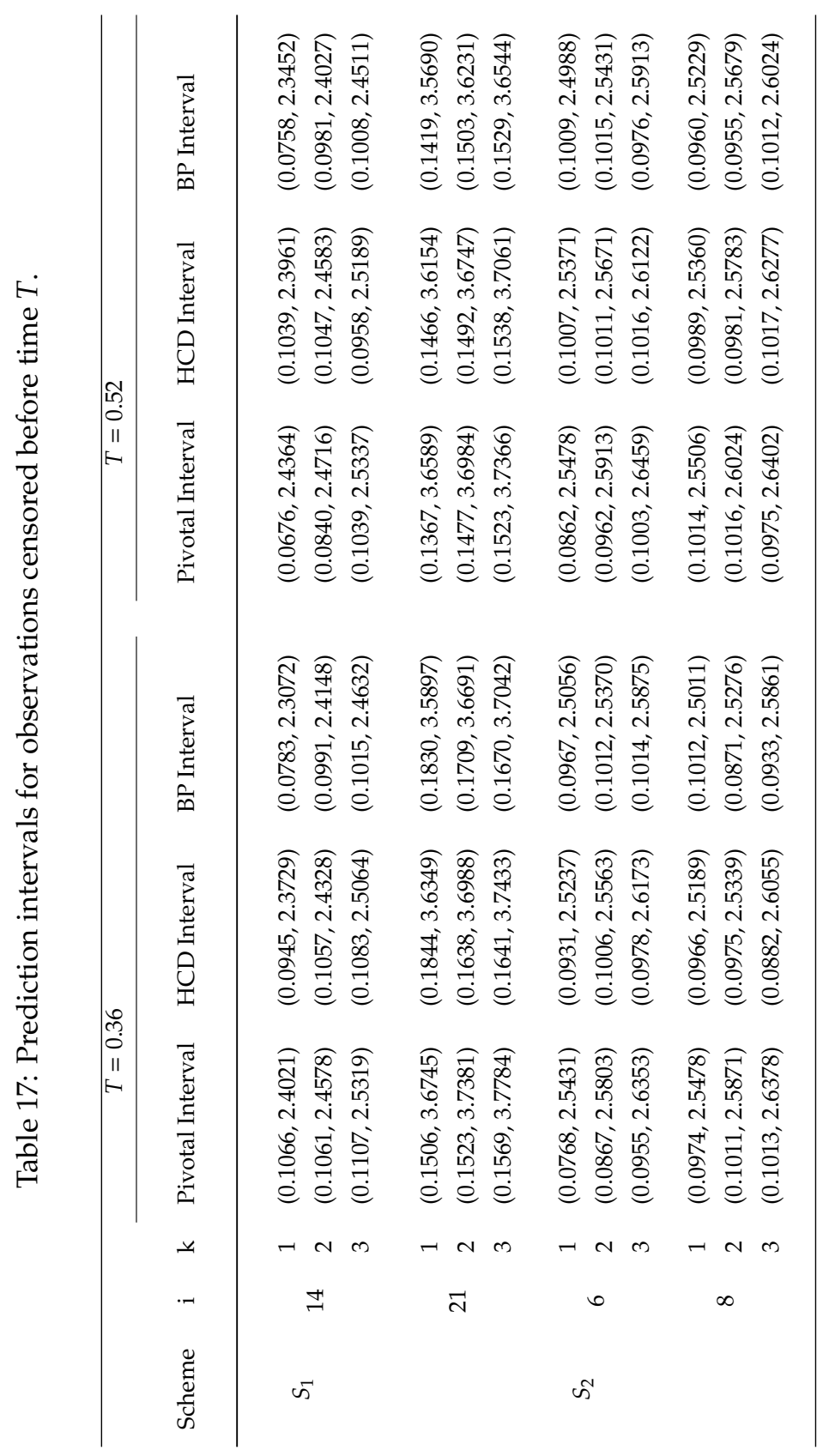


Table 18: Prediction intervals for observations censored after time $T$.

\begin{tabular}{|c|c|c|c|c|c|c|}
\hline Scheme & $T$ & $R_{j}^{*}$ & k & Pivotal Interval & HCD Interval & BP Interval \\
\hline \multirow{2}{*}{$S_{1}$} & \multirow{3}{*}{0.36} & \multirow{3}{*}{41} & 1 & $(0.6571,4.8023)$ & $(0.6633,4.7861)$ & $(0.6339,4.7541)$ \\
\hline & & & 2 & $(0.6566,4.8671)$ & $(0.6829,4.8165)$ & $(0.6544,4.7865)$ \\
\hline \multirow{4}{*}{$S_{1}$} & & & 3 & $(0.6833,4.9035)$ & $(0.6904,4.8641)$ & $(0.6740,4.8349)$ \\
\hline & \multirow{3}{*}{0.52} & \multirow{3}{*}{36} & 1 & $(0.6751,4.8743)$ & $(0.6808,4.8439)$ & $(0.6788,4.8295)$ \\
\hline & & & 2 & $(0.6812,4.9123)$ & $(0.6826,4.8872)$ & $(0.6820,4.8675)$ \\
\hline & & & 3 & $(0.6908,4.9462)$ & $(0.6871,4.9257)$ & $(0.6955,4.9169)$ \\
\hline \multirow{2}{*}{$S_{2}$} & \multirow{3}{*}{0.36} & \multirow{3}{*}{46} & 1 & $(0.7239,4.9876)$ & $(0.7038,4.9655)$ & $(0.7012,4.9440)$ \\
\hline & & & 2 & $(0.7279,5.3217)$ & $(0.7130,5.2631)$ & $(0.7105,5.1973)$ \\
\hline \multirow{4}{*}{$S_{2}$} & & & 3 & $(0.7608,5.5763)$ & $(0.7388,5.5129)$ & $(0.7326,5.4870)$ \\
\hline & \multirow{3}{*}{0.52} & \multirow{3}{*}{43} & 1 & $(0.8011,5.8233)$ & $(0.7819,5.7765)$ & $(0.7791,5.7593)$ \\
\hline & & & 2 & $(0.8244,5.9146)$ & $(0.7905,5.8795)$ & $(0.7857,5.8441)$ \\
\hline & & & 3 & $(0.8270,5.9633)$ & $(0.7881,5.9037)$ & $(0.7877,5.8903)$ \\
\hline
\end{tabular}




\section{Conclusions}

In this paper, we have considered making statistical inferences using the generalized half-normal distribution under progressive hybrid censoring. Cooray and Ananda (2008) initially studied this distribution in literature. Its hazard rate function can be increasing, decreasing and bathtub-shaped depending upon the range of parameters $\alpha$ and $\theta$. Note that some well known distributions such as lognormal, Weibull, generalized exponential, Burr models are useful in situations where the phenomena under study usually indicate monotone hazard rate behavior. Thus generalized half-normal distribution can be treated as a more flexible lifetime model. Materials like polymers, ceramic, glass, gold presented fatigue fracture properties and the generalized halfnormal distribution provides a reasonably adequate fitting to such phenomena. More generally this distribution is suitable for fitting both positively as well as negatively skewed lifetime data. Thus the generalized half-normal distribution is a useful flexible addition to the existing literature of parametric distributions in life testing experiments. At recent past, the family of half-normal distribution has attracted attention of several researchers. Olmos et al. (2012) introduced an elegant extension of the half-normal distribution using the ratio of a half-normal and power of a uniform $U(0,1)$ distribution. They studied various structural properties of the newly proposed model and found it to be useful in fitting a variety of positively skewed reliability data. Through some numerical illustrations, authors concluded that the proposed model provides relatively better fitting than the corresponding half-normal distribution. Further Gmez and Vidal (2016) independently introduced another extension of the half-normal distribution and studied several probabilistic properties of the proposed model. The corresponding hazard rate function can be decreasing or increasing depending upon the range of parameters. This distribution is also useful in analyzing various reliability data. Seemingly the generalized half-normal distribution is more flexible in nature than these two lifetime models. However more work is required to study the effect of these half-normal generalizations in life testing experiments.

Following these discussions we have considered estimation and prediction problems for the generalized half normal distribution using classical and Bayesian approaches when the underlying samples are progressive hybrid censored. We observed that the associated likelihood equation of $\alpha$ and $\theta$ are highly nonlinear in nature. The corresponding MLEs can be computed by simultaneously solving two nonlinear equations using the Newton-Raphson method. However, Pradhan and Kundu (2009) observed that this procedure may not converge always. In such situations, one can apply the EM algorithm to compute MLEs of the unknown parameters. In this case, we 
need to solve one nonlinear equation at a time and so relatively less computations are involved. Further, Fisher information matrix can easily be computed using the missing information principle which is quite useful for constructing asymptotic intervals of the parameters. Similarly, we observed that the posterior distribution of $\alpha$ and $\theta$ given the progressive hybrid censored data are analytically intractable and so we obtained the desired Bayes estimates using known approximation methods. We have also obtained the predictive estimates of censored observations under progressive hybrid censoring and found that proposed classical and the Bayesian predictors work quite good under the given scheme. In the near future, we would like to make some more Bayesian inference for the considered problem using different approximation methods. Largely we have obtained useful inferences for the generalized half-normal distribution and the results are based on numerical computations. Similar observations are also reported in Tomer and Panwar (2015), Hemmati and Khorram (2013). Not much work has been done on this problem under a theoretical framework. We will try to work on it in the near future.

\section{Acknowledgements}

The authors are thankful to a reviewer for valuable suggestions which have significantly improved the content and the presentation of our paper. They also thank the Editor and an Associate Editor for encouraging comments.

\section{References}

Ahmadi, K., Rezaei, M. and Yousefzadeh, F. (2015), Estimation for the generalized half-normal distribution based on progressive type-II censoring. Journal of Statistical Computation and Simulation, 85(6), 1128-1150.

Ahmadi, K. and Yousefzadeh, F. (2015), Estimation for the parameters of generalized half-normal distribution based on progressive type-I interval censoring. Communications in Statistics: Simulation and Computation, 44(10), 2671-2695.

Alma, G. Ö. and Belaghi, R. A. (2016), On the estimation of the extreme value and normal distribution parameters based on progressive type-II hybrid-censored data. Journal of Statistical Computation and Simulation, 86(3), 569-596. 
Andrews, D. F. and Herzberg, A. M. (1985), Data: A Collection of Problems from Many Fields for the Student and Research Worker. New York, Springer Series in Statistics.

Azizpour, M. and Asgharzadeh, A. (2017), Inference for the Type-II generalized logistic distribution with progressive hybrid censoring. Journal of Statistical Research of Iran, 14, 189-217.

Barlow, R. E., Toland, R. H. and Freeman, T. A. (1984), Bayesian analysis of stressrupture life of kevlar 49/epoxy spherical pressure vessels. In: Proc. Canad. Conf. Appl. Statist. New York: Marcel Dekker.

Balakrishnan, N. and Aggarwala, R. (2000), Progressive Censoring: Theory, Methods and Applications. Birkhäuser, Boston.

Balakrishnan, N. and Kundu, D. (2013), Hybrid censoring: models, inferential results and applications (with discussion). Computational Statistics $\mathcal{E}$ Data Analysis, 57(1), 166-209.

Balakrishnan, N. and Cramer, E. (2014), The Art of Progressive Censoring: Applications to Reliability and Quality. Springer, New York.

Cooray, K. and Ananda, M. A. (2008), A generalization of the half-normal distribution with applications to lifetime data. Communications in Statistics: Theory and Methods, 37(9), 1323-1337.

Chen, M. H. and Shao, Q. M. (1999), Monte Carlo estimation of Bayesian credible and HPD intervals. Journal of Computational and Graphical Statistics, 8(1), 69-92.

Dempster, A. P., Laird, N. M. and Rubin, D. B. (1977), Maximum likelihood from incomplete data via the EM algorithm. Journal of the Royal Statistical Society: Series B (methodological), 39, 1-38.

Epstein, B. (1954), Truncated life tests in the exponential case. Annals of Mathematical Statistics, 25(3), 555-564.

Gmez, Y. M. and Vidal, I. (2016), A generalization of the half-normal distribution. Applied Mathematics-A Journal of Chinese Universities, 31(4), 409-424.

Hemmati, F. and Khorram, E. (2013), Statistical analysis of the log-normal distribution under type-II progressive hybrid censoring schemes. Communications in Statistics: Simulation and Computation, 42(1), 52-75. 
Kayal, T., Tripathi, Y. M., Rastogi, M. K. and Asgharzadeh, A. (2017), Inference for Burr XII distribution under type-I progressive hybrid censoring. Communications in Statistics: Simulation and Computation, 46(9), 7447-7465.

Kundu, D. and Joarder, A. (2006), Analysis of type-II progressively hybrid censored data. Computational Statistics and Data Analysis, 50(10), 2509-2528.

Louis, T. A. (1982), Finding the observed information matrix when using the EM algorithm. Journal of the Royal Statistical Society. Series B (Methodological), 44, 226-233.

Lin, C. T., Ng, H. K. T. and Chan, P. S. (2009), Statistical inference of type-II progressively hybrid censored data with Weibull lifetimes. Communications in Statistics: Theory and Methods, 38(10), 1710-1729.

Mokhtari, E. B., Rad, A. H. and Yousefzadeh, F. (2011), Inference for Weibull based on progressively type-II hybrid censored data. Journal of Statistical Planning and Inference, 141(8), 2824-2838.

Olmos, N. M., Varela, H., Gómez, H. W. and Bolfarine, H. (2012), An extension of the half-normal distribution. Statistical Papers, 53(4), 875-886.

Pradhan, B. and Kundu, D. (2009), On progressively censored generalized exponential distribution. Test 18(3), 497-515.

Tierney, L. and Kadane, J. (1986), Accurate approximations for posterior moments and marginals. Journal of American Statistical Association, 81(393), 82-86.

Tomer, S. K. and Panwar, M. S. (2015), Estimation procedures for Maxwell distribution under type-I progressive hybrid censoring scheme. Journal of Statistical Computation and Simulation, 85(2), 339-356. 